\title{
Effective formulas for complex geodesics in generalized pseudoellipsoids with applications
}

\author{
by WŁODZimierz ZwoneK (Kraków)
}

\begin{abstract}
We introduce a class of generalized pseudoellipsoids and we get formulas for their complex geodesics in the convex case. Using these formulas we get a description of automorphisms of the pseudoellipsoids. We also solve the problem of biholomorphic equivalence of convex complex ellipsoids without any sophisticated machinery.
\end{abstract}

1. Introduction. For any domain $D \subset \mathbb{C}^{n}, w, z \in D$ we define

$\widetilde{k}_{D}(w, z)=\inf \left\{p\left(\lambda_{1}, \lambda_{2}\right):\right.$ there is a holomorphic mapping $\varphi: E \rightarrow D$ such that $\left.\varphi\left(\lambda_{1}\right)=w, \varphi\left(\lambda_{2}\right)=z\right\}$,

$c_{D}(w, z)=\sup \{p(\varphi(w), \varphi(z)): \varphi: D \rightarrow E$ is a holomorphic mapping $\}$,

$k_{D}$ is the largest pseudodistance on D not exceeding $\widetilde{k}_{D}$,

where $E$ is the unit open disk in $\mathbb{C}$ and $p$ is the Poincaré distance on $E$.

$\widetilde{k}_{D}$ is called the Lempert function, and $c_{D}$ (respectively $k_{D}$ ) is called the Carathéodory (respectively Kobayashi) pseudodistance of $D$.

Since $\widetilde{k}_{D}, k_{D}, c_{D}$ are often not very easy to handle we shall often use the associated functions with the same properties: $d_{D}^{*}=\tanh \left(d_{D}\right)$, where $d$ is $\widetilde{k}, k$ or $c$.

Below writing $d$ we mean $c, k$ or $\widetilde{k}$.

Definition 1.1. For a domain $D$ we say that a mapping $\varphi: E \rightarrow D$ is a $d$-geodesic for $D$ if $d_{D}\left(\varphi\left(\lambda_{1}\right), \varphi\left(\lambda_{2}\right)\right)=p\left(\lambda_{1}, \lambda_{2}\right)$ for any $\lambda_{1}, \lambda_{2} \in E$.

It is known that for any domain $D \subset \mathbb{C}^{n}$,

$$
c_{D} \leq k_{D} \leq \widetilde{k}_{D}
$$

If $D$ is a convex domain, then we have equality:

1991 Mathematics Subject Classification: Primary 32H15.

Key words and phrases: complex geodesics, generalized pseudoellipsoids, biholomorphic equivalence of ellipsoids. 
Theorem 1.2 (see $[\mathrm{L}])$. If $D \subset \mathbb{C}^{n}$ is a convex domain, then

$$
c_{D}=\widetilde{k}_{D} .
$$

Moreover, if $D$ is additionally bounded, then for all $(w, z) \in D \times D$ with $w \neq z$ there is a $k$-geodesic $\varphi: E \rightarrow D$ such that $w, z \in \varphi(E)$ (certainly this mapping is also a $\widetilde{k}$ - and c-geodesic).

In view of Theorem 1.2 we can introduce the notion of a complex geodesic for convex domains because all the geodesics that we consider are in this case identical.

Below we define a class of domains, which are in the scope of our interest. Fix $n \in \mathbb{N}_{*}$. Assume that $m_{0}$ is a positive natural number; for $j=1, \ldots, m_{0}$ consider numbers $m_{j} \in \mathbb{N}_{*}$. Next, we assume that we have positive natural numbers $\left\{m_{j, k}\right\}_{k=1}^{m_{j}}$, where $j=1, \ldots, m_{0}$; and, generally, for $k \leq n-1$ we have $\left\{m_{j_{1}, \ldots, j_{k}}\right\}_{j_{k}=1}^{m_{j_{1}, \ldots, j_{k-1}}} \subset \mathbb{N}_{*}$. Given a system of $m$ 's we consider a system of positive numbers given as follows: $\left\{p_{j}\right\}_{j=1}^{m_{0}}$, $\left\{p_{j, k}\right\}_{k=1}^{m_{j}}$, where $j=1, \ldots, m_{0}$; and, generally, $\left\{p_{j_{1}, \ldots, j_{k}}\right\}_{j_{k}=1}^{m_{j_{1}, \ldots, j_{k-1}}}$, where $k=1, \ldots, n-1$.

Let us define a generalized complex pseudoellipsoid $\mathcal{E}$ by

$$
\mathcal{E}:=\left\{z: \sum_{j_{1}=1}^{m_{0}}\left(\ldots\left(\sum_{j_{n}=1}^{m_{j_{1}, \ldots, j_{n-1}}}\left|z_{j_{1}, \ldots, j_{n}}\right|^{2}\right)^{p_{j_{1}}, \ldots, j_{n-1}} \ldots\right)^{p_{j_{1}}}<1\right\},
$$

where $m$ 's and $p$ 's are as above.

Let us introduce the following convention: if in the sequel we have some letter with a system of subscripts $\left(j_{1}, \ldots, j_{k}\right)$, where $k=0$, then we mean the same letter with subscript 0 . For a point $z \in \mathbb{C}^{N}$, where

$$
N=\sum_{j_{1}=1}^{m_{0}}\left(\ldots\left(\sum_{j_{n-1}=1}^{m_{j_{1}, \ldots, j_{n-2}}} m_{j_{1}, \ldots, j_{n-1}}\right) \ldots\right),
$$

writing $z=\left(z_{1}, \ldots, z_{m_{0}}\right)$ we mean $z_{j}=\left(z_{j, 1}, \ldots, z_{j, m_{j}}\right)$ for $j=1, \ldots, m_{0}$

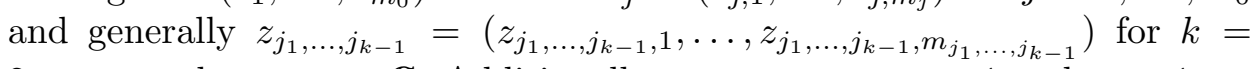
$2, \ldots, n$ and $z_{j_{1}, \ldots, j_{n}} \in \mathbb{C}$. Additionally, we put $p_{j_{1}, \ldots, j_{n}}:=1$ and $p_{0}=1$.

We also use the same convention for mappings going to $\mathbb{C}^{N}$. For convenience set, for $k=1, \ldots, n$ and fixed $\left(j_{1}, \ldots, j_{k-1}\right)$,

$$
\begin{aligned}
& J_{j_{1}, \ldots, j_{k-1}}(z) \\
& \quad:=\sum_{j_{k}=1}^{m_{j_{1}, \ldots, j_{k-1}}}\left(\ldots\left(\sum_{j_{n}=1}^{m_{j_{1}}, \ldots, j_{n-1}}\left|z_{j_{1}, \ldots, j_{n}}\right|^{2}\right)^{p_{j_{1}, \ldots, j_{n-1}}} \ldots\right)^{p_{j_{1}, \ldots, j_{k}}},
\end{aligned}
$$


for $z \in \mathbb{C}^{N}$. Certainly,

$$
J_{j_{1}, \ldots, j_{k-1}}(z)=\sum_{j_{k}=1}^{m_{j_{1}, \ldots, j_{k-1}}} J_{j_{1}, \ldots, j_{k}}(z)^{p_{j_{1}, \ldots, j_{k}}} .
$$

Note that for $n=1, \mathcal{E}$ is an $m_{0}$-dimensional Euclidean ball, which we denote by $B_{m_{0}}$. If $n=2$, then $\mathcal{E}$ is a generalized complex ellipsoid considered for instance in [KKM] and [DP] (if $m_{j}=1$ for $j=1, \ldots, m_{0}$, then we have usual complex ellipsoids).

Below we only consider pseudoellipsoids such that

$$
p_{j_{1}, \ldots, j_{n}} \ldots p_{j_{1}, \ldots, j_{k}} \geq 1 / 2
$$

for $k=1, \ldots, n-1$ and all possible $\left(j_{1}, \ldots, j_{n}\right)$.

LEMma 1.3. A generalized pseudoellipsoid $\mathcal{E}$ is convex iff $\mathcal{E}$ may be defined by coefficients $p_{j_{1}, \ldots, j_{k}}$ satisfying (1.3).

Proof. To prove sufficiency we verify that for $j_{1}=1, \ldots, m_{0}$ the function $J_{j_{1}}^{p_{j_{1}}}$ is convex on $\mathbb{C}^{N}$.

Using induction on $k$ we prove that $J_{j_{1}, \ldots, j_{k}}^{p_{j_{1}, \ldots, j_{k}}}$ is convex for all $k,\left(j_{1}, \ldots\right.$ $\left.\ldots, j_{k}\right)$ and any system of $p_{j_{1}, \ldots, j_{t}}$ with $t=n, \ldots, k$ satisfying (1.3).

If $k=n-1$, then

$$
J_{j_{1}, \ldots, j_{n-1}}^{p_{j_{1}, \ldots, j_{n-1}}}(z)=\left\|z_{j_{1}, \ldots, j_{n-1}}\right\|^{2 p_{j_{1}, \ldots, j_{n-1}}},
$$

where the exponent is at least 1 , which completes the first inductive step.

Assume that we have proved convexity for $k, 2 \leq k \leq n-1$, and consider the case $k-1$. For $t \in[0,1]$ we have

$J_{j_{1}, \ldots, j_{k-1}}^{p_{j_{1}}, \ldots, j_{k-1}}(t z+(1-t) w)=\left(\sum_{j_{k}=1}^{m_{j_{1}, \ldots, j_{k-1}}} J_{j_{1}, \ldots, j_{k}}^{p_{j_{1}, \ldots, j_{k}}}(t z+(1-t) w)\right)^{p_{j_{1}, \ldots, j_{k-1}}}$.

Let us put

$$
\begin{aligned}
\widetilde{p}_{j_{1}, \ldots, j_{k}} & :=\min _{j_{n}, \ldots, j_{k+1}}\left\{2 p_{j_{1}, \ldots, j_{n}} \ldots p_{j_{1}, \ldots, j_{k+1}}\right\}, \\
\widetilde{p}_{j_{1}, \ldots, j_{k-1}} & :=\min _{j_{n}, \ldots, j_{k}}\left\{2 p_{j_{1}, \ldots, j_{n}} \ldots p_{j_{1}, \ldots, j_{k}}\right\} .
\end{aligned}
$$

Using the inductive assumption for $J_{j_{1}, \ldots, j_{k}}^{1 / \tilde{p}_{j_{1}}, \ldots, j_{k}}$, the fact that $\tilde{p}_{j_{1}, \ldots, j_{k}} p_{j_{1}, \ldots, j_{k}} /$ $\widetilde{p}_{j_{1}, \ldots, j_{k-1}}$ and $\tilde{p}_{j_{1}, \ldots, j_{k-1}} p_{j_{1}, \ldots, j_{k-1}}$ are at least 1 (so suitable functions are convex) and the triangle inequality for the norm $\left\|\left(x_{1}, \ldots, x_{s}\right)\right\|_{q}:=$ $\left(\left|x_{1}\right|^{q}+\ldots+\left|x_{s}\right|^{q}\right)^{1 / q}$, where $s=m_{j_{1}, \ldots, j_{k-1}}, q=\widetilde{p}_{j_{1}, \ldots, j_{k-1}}(q$ is at least 1$)$ 
we get

$$
\begin{aligned}
& \left(\sum_{j_{k}=1}^{m_{j_{1}, \ldots, j_{k-1}}} J_{j_{1}, \ldots, j_{k}}^{\frac{1}{\tilde{p}_{j_{1}}, \ldots, j_{k}}} \tilde{p}_{j_{1}, \ldots, j_{k}} p_{j_{1}, \ldots, j_{k}}(t z+(1-t) w)\right)^{p_{j_{1}, \ldots, j_{k}-1}} \\
& \leq\left(\left(\sum _ { j _ { k } = 1 } ^ { m _ { j _ { 1 } , \ldots , j _ { k - 1 } } } \left(\left(t J_{j_{1}, \ldots, j_{k}}^{\tilde{\tilde{p}}_{j_{1}, \ldots, j_{k}}}(z)+(1-t)\right.\right.\right.\right. \\
& \left.\left.\left.\left.\times J_{j_{1}, \ldots, j_{k}}^{\frac{1}{\tilde{\tilde{p}}_{j_{1}, \ldots, j_{k}}}}(w)\right)^{\frac{\tilde{p}_{j_{1}, \ldots, j_{k}} p_{j_{1}, \ldots, j_{k}}}{\tilde{p}_{j_{1}, \ldots, j_{k-1}}}}\right)^{\tilde{p}_{j_{1}, \ldots, j_{k-1}}}\right)^{\frac{1}{\tilde{p}_{j_{1}, \ldots, j_{k-1}}}}\right)^{\tilde{p}_{j_{1}, \ldots, j_{k-1}} p_{j_{1}, \ldots, j_{k-1}}} \\
& \leq\left(\left(\sum _ { j _ { k } = 1 } ^ { m _ { j _ { 1 } , \ldots , j _ { k - 1 } } } \left(t J_{j_{1}, \ldots, j_{k}}^{\frac{p_{j_{1}, \ldots, j_{k}}}{\tilde{p}_{j_{1}, \ldots, j_{k}-1}}}(z)\right.\right.\right. \\
& \left.\left.\left.+(1-t) J_{j_{1}, \ldots, j_{k}}^{\frac{p_{j_{1}}, \ldots, j_{k}}{\tilde{p}_{j_{1}}, \ldots, j_{k-1}}}(w)\right)^{\tilde{p}_{j_{1}, \ldots, j_{k-1}}}\right)^{\frac{1}{\tilde{p}_{j_{1}, \ldots, j_{k-1}}}}\right)^{\tilde{p}_{j_{1}, \ldots, j_{k-1}} p_{j_{1}, \ldots, j_{k-1}}} \\
& \leq\left(t\left(\sum_{j_{k}=1}^{m_{j_{1}, \ldots, j_{k-1}}} J_{j_{1}, \ldots, j_{k}}^{p_{j_{1}, \ldots, j_{k}}}(z)\right)^{\frac{1}{\tilde{p}_{j_{1}, \ldots, j_{k-1}}}}\right. \\
& \left.+(1-t)\left(\sum_{j_{k}=1}^{m_{j_{1}, \ldots, j_{k-1}}} J_{j_{1}, \ldots, j_{k}}^{p_{j_{1}, \ldots, j_{k}}}(w)\right)^{\frac{1}{\tilde{p}_{j_{1}, \ldots, j_{k-1}}}}\right)^{\tilde{p}_{j_{1}, \ldots, j_{k-1}} p_{j_{1}, \ldots, j_{k-1}}} \\
& \leq t J_{j_{1}, \ldots, j_{k-1}}^{p_{j_{1}, \ldots, j_{k-1}}}(z)+(1-t) J_{j_{1}, \ldots, j_{k-1}}^{p_{j_{1}}, \ldots, j_{k-1}}(w)
\end{aligned}
$$

which completes the proof of sufficiency.

Suppose now that $\mathcal{E}$ is convex and cannot be defined by $p$ 's satisfying (1.3). Consequently, we can find (changing $p$ 's if necessary) $k \in\{1, \ldots, n-1\}$ and $\left(j_{1}, \ldots, j_{n}\right)$ such that

$$
p_{\jmath_{1}, \ldots, \jmath_{n}} \ldots p_{\jmath_{1}, \ldots, j_{k}}<1 / 2 \text { and } m_{\jmath_{1}, \ldots, \jmath_{k-1}}>1 \text {. }
$$

There is a subscript $\left(\jmath_{1}, \ldots, \stackrel{\jmath}{j-1}_{k-1}, j_{k}, \ldots, j_{n}\right)$ of $p$, where $j_{k} \neq \jmath_{k}$. Note that the intersection of the linear subspace spanned by the $\left(\rho_{1}, \ldots, j_{n}\right)$ th and the $\left(\jmath_{1}, \ldots, \jmath_{k-1}, j_{k}, \ldots, j_{n}\right)$ th vectors from the standard vector base of $\mathbb{C}^{N}$ with $\mathcal{E}$ is linearly isomorphic to

$$
\left\{\left(\lambda_{1}, \lambda_{2}\right) \in \mathbb{C}^{2}:\left|\lambda_{1}\right|^{2 q_{1}}+\left|\lambda_{2}\right|^{2 q_{2}}<1\right\},
$$

where $q_{1}=p_{\jmath_{1}, \ldots, \jmath_{n}} \ldots p_{\jmath_{1}, \ldots, \jmath_{k}}, q_{2}=p_{\jmath_{1}, \ldots, \jmath_{k-1}, j_{k}, \ldots, j_{n}} \ldots p_{\jmath_{1}, \ldots, \jmath_{k-1}, j_{k}}$, which is not convex - a contradiction.

In view of Lemma 1.3, for generalized pseudoellipsoids with (1.3) we may consider complex geodesics. In the sequel, unless otherwise stated, we always assume that $\mathcal{E}$ satisfies (1.3). 
In Chapter 2 we formulate and prove a theorem giving explicit formulas for complex geodesics in convex generalized pseudoellipsoids. The idea of the proof is identical to the one presented in [JPZ] (see also [JP]), where these formulas are obtained for convex complex ellipsoids. Since, however, some parts of the proof are a little more subtle and tedious than those presented in the above mentioned works we give the whole proof. Let us remark here that so far very few effective formulas for complex geodesics are known. Besides the above mentioned works let us mention the papers [BFKKMP], $[\mathrm{P}],[\mathrm{Ge}],[\mathrm{DT}]$ dealing with formulas for complex geodesics in special cases of convex ellipsoids.

In Chapter 3 we prove a proposition which allows us to describe all automorphisms of $\mathcal{E}$ (however, not for all convex $\mathcal{E}$ ). The proof is based on the explicit formulas for complex geodesics and an extension theorem for biholomorphic mappings between bounded complete Reinhardt domains (see $[\mathrm{JP}],[\mathrm{B}]$ ).

Finally, in Chapter 4, we restrict our attention to a special case of $\mathcal{E}$ and we solve the problem of biholomorphic equivalence of convex complex ellipsoids by only using the formulas for complex geodesics but avoiding the use of the theorem on holomorphic extension of biholomorphic mappings between ellipsoids to their closures. This gives an answer to a question in $[\mathrm{JP}]$. Let us underline once more that in this proof we avoid the use of the theory of the Bergman kernel (as in $[\mathrm{JP}]$ ) or Lie theory (as in $[\mathrm{KU}]$ and $[\mathrm{N}]$ ).

2. Explicit formulas for convex generalized pseudoellipsoids. As already announced we assume in this chapter that $\mathcal{E}$ satisfies condition (1.3).

LEMMA 2.1. Let $\varphi: E \rightarrow \mathbb{C}^{N}$ be a bounded, nonconstant, holomorphic mapping such that $\varphi_{j_{1}, \ldots, j_{n}} \not \equiv 0$ for all possible $\left(j_{1}, \ldots, j_{n}\right)$. Then $\varphi$ is a geodesic in $\mathcal{E}$ iff there are mappings $h_{j_{1}, \ldots, j_{n}} \in H^{1}(E, \mathbb{C})$ and $\varrho: \partial E \rightarrow \mathbb{R}_{>0}$ such that

$$
\begin{aligned}
& \frac{1}{\lambda} h_{j_{1}, \ldots, j_{n}}^{*}(\lambda) \\
= & \varrho(\lambda)\left(\prod_{k=2}^{n} p_{j_{1}, \ldots, j_{k-1}}\left(J_{j_{1}, \ldots, j_{k-1}}\left(\varphi^{*}(\lambda)\right)\right)^{p_{j_{1}, \ldots, j_{k-1}}-1}\right) \frac{\varphi_{j_{1}, \ldots, j_{n}}^{*}(\lambda)}{\text { a.e. on } \partial E} \\
J_{0}\left(\varphi^{*}(\lambda)\right)=1 & \text { a.e. on } \partial E
\end{aligned}
$$

with the product equal to 1 if $n=1$.

Here $H^{1}$ denotes the Hardy space, $\varphi_{j_{1}, \ldots, j_{n}}^{*}$ and $h_{j_{1}, \ldots, j_{n}}^{*}$ stand for nontangential boundary values. 
Proof. This follows from the fact that the unit outer normal vector $\nu(z) \in \mathbb{C}^{N}$ to $\partial \mathcal{E}$ at $z \in \partial \mathcal{E} \cap\left(\mathbb{C}_{*}\right)^{N}$ is given by the formula

$$
\nu_{j_{1}, \ldots, j_{n}}(z)=\widetilde{\varrho}(z)\left(\prod_{k=2}^{n} p_{j_{1}, \ldots, j_{k-1}}\left(J_{j_{1}, \ldots, j_{k-1}}(z)\right)^{p_{j_{1}, \ldots, j_{k-1}}-1}\right) z_{j_{1}, \ldots, j_{n}},
$$

with the product equal to 1 if $n=1$, where $\widetilde{\varrho}(z)>0$, and from Corollary 8.4 .5 of $[\mathrm{JP}]$.

ThEOREM 2.2. A bounded holomorphic mapping $\varphi: E \rightarrow \mathbb{C}^{N}$ such that $\varphi_{j_{1}, \ldots, j_{n}} \not \equiv 0$ for all possible $\left(j_{1}, \ldots, j_{n}\right)$ is a geodesic in $\mathcal{E}$ iff

$$
\begin{aligned}
\varphi_{j_{1}, \ldots, j_{n}}(\lambda)= & \left(\frac{\lambda-\alpha_{j_{1}, \ldots, j_{n}}}{1-\bar{\alpha}_{j_{1}, \ldots, j_{n}} \lambda}\right)^{s_{j_{1}, \ldots, j_{n}}} \\
& \times\left(\prod_{k=1}^{n}\left(a_{j_{1}, \ldots, j_{k}}\left(\frac{1-\bar{\alpha}_{j_{1}, \ldots, j_{k}} \lambda}{1-\bar{\alpha}_{j_{1}, \ldots, j_{k-1}} \lambda}\right)\right)^{\frac{1}{p_{j_{1}, \ldots, j_{k}} \ldots p_{j_{1}, \ldots, j_{n}}}}\right),
\end{aligned}
$$

where $s_{j_{1}, \ldots, j_{n}} \in\{0,1\}, a_{j_{1}, \ldots, j_{k}} \in \mathbb{C}_{*}, \alpha_{j_{1}, \ldots, j_{k}} \in \bar{E}$ for all possible $\left(j_{1}, \ldots\right.$ $\left.\ldots, j_{k}\right), \alpha_{0} \in E$; if additionally $s_{j_{1}, \ldots, j_{n}}=1$, then $\alpha_{j_{1}, \ldots, j_{n}} \in E$; moreover if $\left|\alpha_{j_{1}, \ldots, j_{k-1}}\right|=1$, then $\alpha_{j_{1}, \ldots, j_{k}}=\alpha_{j_{1}, \ldots, j_{k-1}}$ for all $j_{k}=1, \ldots, m_{j_{1}, \ldots, j_{k-1}}$; also, the following relations hold for $k=1, \ldots, n$ :

$$
\begin{aligned}
\alpha_{j_{1}, \ldots, j_{k-1}} & =\sum_{j_{k}=1}^{m_{j_{1}, \ldots, j_{k-1}}}\left|a_{j_{1}, \ldots, j_{k}}\right|^{2} \alpha_{j_{1}, \ldots, j_{k}}, \\
1+\left|\alpha_{j_{1}, \ldots, j_{k-1}}\right|^{2} & =\sum_{j_{k}=1}^{m_{j_{1}, \ldots, j_{k-1}}}\left|a_{j_{1}, \ldots, j_{k}}\right|^{2}\left(1+\left|\alpha_{j_{1}, \ldots, j_{k}}\right|^{2}\right) .
\end{aligned}
$$

Finally, the case $s_{j_{1}, \ldots, j_{n}}=0$ and $\alpha_{j_{1}, \ldots, j_{k}}=\alpha_{0}$ for all possible $\left(j_{1}, \ldots, j_{k}\right)$ is excluded.

Proof. First we prove that the above formulas are really formulas for complex geodesics. For those $\lambda \in \bar{E}$ for which it makes sense, define

$$
P_{j_{1}, \ldots, j_{n}, l}(\lambda):=\prod_{k=1}^{l}\left(a_{j_{1}, \ldots, j_{k}}\left(\frac{1-\bar{\alpha}_{j_{1}, \ldots, j_{k}} \lambda}{1-\bar{\alpha}_{j_{1}, \ldots, j_{k-1}} \lambda}\right)\right)^{\frac{1}{p_{j_{1}, \ldots, j_{k} \cdots p_{j_{1}, \ldots, j_{n}}}}}
$$

for $l=1, \ldots, n$ and, additionally, $P_{j_{1}, \ldots, j_{n}, 0}(\lambda):=1$.

We also put

$$
Q_{j_{1}, \ldots, j_{l}}:=P_{j_{1}, \ldots, j_{n}, l}^{p_{j_{1}, \ldots, j_{n}} \ldots p_{j_{1}, \ldots, j_{l+1}}} \quad \text { for } l=0, \ldots, n-1 .
$$


Since the right-hand side does not depend on the choice of $\left(j_{l+1}, \ldots, j_{n}\right)$, $Q_{j_{1}, \ldots, j_{l}}$ is well defined.

We have the following equality a.e. on $\partial E$ :

$$
\begin{aligned}
J_{j_{1}, \ldots, j_{n-1}}\left(\varphi^{*}(\lambda)\right) & =\sum_{j_{n}=1}^{m_{j_{1}, \ldots, j_{n-1}}}\left|\varphi_{j_{1}, \ldots, j_{n}}^{*}(\lambda)\right|^{2} \\
& =\left|Q_{j_{1}, \ldots, j_{n-1}}(\lambda)\right|^{2} \sum_{j_{n}=1}^{m_{j_{1}, \ldots, j_{n-1}}}\left|a_{j_{1}, \ldots, j_{n}}\right|^{2} \frac{\left|1-\bar{\alpha}_{j_{1}, \ldots, j_{n}} \lambda\right|^{2}}{\left|1-\bar{\alpha}_{j_{1}, \ldots, j_{n-1}} \lambda\right|^{2}},
\end{aligned}
$$

which in view of (2.3) and (2.4) equals $\left|Q_{j_{1}, \ldots, j_{n-1}}(\lambda)\right|^{2}$. For the same reason, for almost all $\lambda \in \partial E$ we have

$$
\begin{aligned}
J_{j_{1}, \ldots, j_{n-2}}\left(\varphi^{*}(\lambda)\right) & \\
= & \sum_{j_{n-1}=1}^{m_{j_{1}, \ldots, j_{n-2}}}\left(J_{j_{1}, \ldots, j_{n-1}}\left(\varphi^{*}(\lambda)\right)\right)^{p_{j_{1}, \ldots, j_{n-1}}} \\
& =\sum_{j_{n-1}=1}^{m_{j_{1}, \ldots, j_{n-2}}}\left|Q_{j_{1}, \ldots, j_{n-1}}(\lambda)\right|^{2 p_{j_{1}, \ldots, j_{n-1}}} \\
& =\left|Q_{j_{1}, \ldots, j_{n-2}}(\lambda)\right|^{2} \sum_{j_{n-1}=1}^{m_{j_{1}, \ldots, j_{n-2}}}\left|a_{j_{1}, \ldots, j_{n-1}}\right|^{2} \frac{\left|1-\bar{\alpha}_{j_{1}, \ldots, j_{n-1}} \lambda\right|^{2}}{\left|1-\bar{\alpha}_{j_{1}, \ldots, j_{n-2}} \lambda\right|^{2}} \\
& =\left|Q_{j_{1}, \ldots, j_{n-2}}(\lambda)\right|^{2},
\end{aligned}
$$

and generally, for almost all $\lambda \in \partial E$,

$$
J_{j_{1}, \ldots, j_{l-1}}\left(\varphi^{*}(\lambda)\right)=\left|Q_{j_{1}, \ldots, j_{l-1}}(\lambda)\right|^{2}
$$

for $l=1, \ldots, n$. For $l=1$ we thus get (2.2). To prove (2.1), define

$$
\begin{aligned}
h_{j_{1}, \ldots, j_{n}}(\lambda):= & \frac{\left(1-\bar{\alpha}_{j_{1}, \ldots, j_{n}} \lambda\right)^{2}}{P_{j_{1}, \ldots, j_{n}, n}(\lambda)} \\
& \times\left(\frac{\lambda-\alpha_{j_{1}, \ldots, j_{n}}}{1-\bar{\alpha}_{j_{1}, \ldots, j_{n-1}} \lambda}\right)^{1-s_{j_{1}, \ldots, j_{n}}} \prod_{k=1}^{n} p_{j_{1}, \ldots, j_{k}}\left|a_{j_{1}, \ldots, j_{k}}\right|^{2}
\end{aligned}
$$

for $\lambda \in E$ and

$$
\varrho(\lambda):=\left|1-\alpha_{0} \lambda\right|^{2} \quad \text { for } \lambda \in \partial E .
$$

In view of the assumptions on $\alpha_{j_{1}, \ldots, j_{k}}$ and $(1.3), h_{j_{1}, \ldots, j_{n}} \in H^{\infty}(E)$. We want to prove that a.e. on $\partial E$ we have 


$$
\begin{aligned}
\frac{1}{\lambda}\left(1-\bar{\alpha}_{j_{1}, \ldots, j_{n}} \lambda\right)^{2} & \left(\frac{\lambda-\alpha_{j_{1}, \ldots, j_{n}}}{1-\bar{\alpha}_{j_{1}, \ldots, j_{n}} \lambda}\right)^{1-s_{j_{1}, \ldots, j_{n}}} \prod_{k=1}^{n} p_{j_{1}, \ldots, j_{k}}\left|a_{j_{1}, \ldots, j_{k}}\right|^{2} \\
= & \varrho(\lambda)\left(\prod_{k=2}^{n} p_{j_{1}, \ldots, j_{k-1}}\left(J_{j_{1}, \ldots, j_{k-1}}\left(\varphi^{*}(\lambda)\right)\right)^{p_{j_{1}, \ldots, j_{k-1}}-1}\right) \\
& \times \frac{\left(\frac{\lambda-\alpha_{j_{1}, \ldots, j_{n}}}{1-\bar{\alpha}_{j_{1}, \ldots, j_{n}} \lambda}\right)^{s_{j_{1}, \ldots, j_{n}}}\left|P_{j_{1}, \ldots, j_{n}, n}(\lambda)\right|^{2} .}{}
\end{aligned}
$$

By (2.5) this is equivalent to

$$
\begin{aligned}
& \frac{1}{\lambda}\left(1-\bar{\alpha}_{j_{1}, \ldots, j_{n}} \lambda\right)\left(\lambda-\alpha_{j_{1}, \ldots, j_{n}}\right) \prod_{k=1}^{n} p_{j_{1}, \ldots, j_{k}}\left|a_{j_{1}, \ldots, j_{k}}\right|^{2} \\
& =\varrho(\lambda)\left(\prod_{k=2}^{n} p_{j_{1}, \ldots, j_{k-1}}\left|Q_{j_{1}, \ldots, j_{k-1}}(\lambda)\right|^{2\left(p_{j_{1}, \ldots, j_{k-1}}-1\right)}\right)\left|P_{j_{1}, \ldots, j_{n}, n}(\lambda)\right|^{2}
\end{aligned}
$$

But in view of the definition of $P_{j_{1}, \ldots, j_{n}, k}$ and $Q_{j_{1}, \ldots, j_{k}}$ the exponent of the expression

$$
\left|a_{j_{1}, \ldots, j_{k}}\left(\frac{1-\bar{\alpha}_{j_{1}, \ldots, j_{k}} \lambda}{1-\bar{\alpha}_{j_{1}, \ldots, j_{k-1}} \lambda}\right)\right|
$$

for $k=1, \ldots, n-1$ on the right-hand side of $(2.6)$ is

$$
\begin{aligned}
\frac{2}{p_{j_{1}, \ldots, j_{k}} \ldots p_{j_{1}, \ldots, j_{n}}} & +\frac{2\left(p_{j_{1}, \ldots, j_{k}}-1\right)}{p_{j_{1}, \ldots, j_{k}}} \\
& +\frac{2\left(p_{j_{1}, \ldots, j_{k+1}}-1\right)}{p_{j_{1}, \ldots, j_{k}} p_{j_{1}, \ldots, j_{k+1}}}+\ldots+\frac{2\left(p_{j_{1}, \ldots, j_{n-1}}-1\right)}{p_{j_{1}, \ldots, j_{k}} \ldots p_{j_{1}, \ldots, j_{n-1}}},
\end{aligned}
$$

which equals 2 . One can also easily see that the exponent is the same for $k=n$. Therefore, (2.6) reduces to

$$
\frac{1}{\lambda}\left(1-\bar{\alpha}_{j_{1}, \ldots, j_{n}} \lambda\right)\left(\lambda-\alpha_{j_{1}, \ldots, j_{n}}\right)=\left|1-\bar{\alpha}_{0} \lambda\right|^{2} \prod_{k=1}^{n}\left|\frac{1-\bar{\alpha}_{j_{1}, \ldots, j_{k}} \lambda}{1-\bar{\alpha}_{j_{1}, \ldots, j_{k-1}} \lambda}\right|^{2},
$$

i.e.

$$
\frac{1}{\lambda}\left(1-\bar{\alpha}_{j_{1}, \ldots, j_{n}} \lambda\right)\left(\lambda-\alpha_{j_{1}, \ldots, j_{n}}\right)=\left|1-\bar{\alpha}_{j_{1}, \ldots, j_{n}} \lambda\right|^{2},
$$

which is obviously true for $\lambda \in \partial E$.

To prove the converse implication one can take $h_{j_{1}, \ldots, j_{n}}$ as in Lemma 2.1. In view of Lemma 8.4.6 of [JP] (see also [Ge]) we get, for $\lambda \in E$,

$$
\varphi_{j_{1}, \ldots, j_{n}}(\lambda) h_{j_{1}, \ldots, j_{n}}(\lambda)=r_{j_{1}, \ldots, j_{n}}\left(\lambda-\alpha_{j_{1}, \ldots, j_{n}}\right)\left(1-\bar{\alpha}_{j_{1}, \ldots, j_{n}} \lambda\right),
$$




$$
\begin{aligned}
& \sum_{j_{k}=1}^{m_{j_{1}, \ldots, j_{k-1}}} \cdots \sum_{j_{n}=1}^{m_{j_{1}, \ldots, j_{n-1}}} \varphi_{j_{1}, \ldots, j_{n}}(\lambda) h_{j_{1}, \ldots, j_{n}}(\lambda) \\
& \quad=r_{j_{1}, \ldots, j_{k-1}}\left(\lambda-\alpha_{j_{1}, \ldots, j_{k-1}}\right)\left(1-\bar{\alpha}_{j_{1}, \ldots, j_{k-1}} \lambda\right)
\end{aligned}
$$

where $k=1, \ldots, n ; r_{j_{1}, \ldots, j_{p}}>0, \alpha_{j_{1}, \ldots, j_{p}} \in \bar{E}$ for $p=0, \ldots, n$ and all possible $\left(j_{1}, \ldots, j_{p}\right)$; if $\varphi_{j_{1}, \ldots, j_{n}}$ has a root in $E$, then we put $s_{j_{1}, \ldots, j_{n}}:=1$, otherwise $s_{j_{1}, \ldots, j_{n}}:=0$.

We see that if $s_{j_{1}, \ldots, j_{n}}=1$, then $\alpha_{j_{1}, \ldots, j_{n}} \in E$. From (2.7) and (2.8) we get

$$
\begin{gathered}
r_{j_{1}, \ldots, j_{k-1}} \alpha_{j_{1}, \ldots, j_{k-1}}=\sum_{j_{k}=1}^{m_{j_{1}, \ldots, j_{k-1}}} r_{j_{1}, \ldots, j_{k}} \alpha_{j_{1}, \ldots, j_{k}}, \\
r_{j_{1}, \ldots, j_{k-1}}\left(1+\left|\alpha_{j_{1}, \ldots, j_{k-1}}\right|^{2}\right)=\sum_{j_{k}=1}^{m_{j_{1}, \ldots, j_{k-1}}} r_{j_{1}, \ldots, j_{k}}\left(1+\left|\alpha_{j_{1}, \ldots, j_{k}}\right|^{2}\right),
\end{gathered}
$$

for $k=1, \ldots, n$ and all possible $\left(j_{1}, \ldots, j_{k-1}\right)$.

From (2.9) and (2.10) we conclude that a.e. on $\partial E$ we have

$$
\sum_{j_{k}=1}^{m_{j_{1}, \ldots, j_{k-1}}} \frac{r_{j_{1}, \ldots, j_{k}}}{r_{j_{1}, \ldots, j_{k-1}}}\left|\frac{1-\bar{\alpha}_{j_{1}, \ldots, j_{k}} \lambda}{1-\bar{\alpha}_{j_{1}, \ldots, j_{k-1}} \lambda}\right|^{2}=1 .
$$

Hence we easily see that

$(2.12) \quad$ if $\left|\alpha_{j_{1}, \ldots, j_{k-1}}\right|=1$, then $\alpha_{j_{1}, \ldots, j_{k}}=\alpha_{j_{1}, \ldots, j_{k-1}}$

$$
\text { for } j_{k}=1, \ldots, m_{j_{1}, \ldots, j_{k-1}}
$$

in particular,

(2.13) if $\left|\alpha_{0}\right|=1$, then $\alpha_{j_{1}, \ldots, j_{p}}=\alpha_{0}$

$$
\text { for } p=1, \ldots, n \text { and all possible }\left(j_{1}, \ldots, j_{p}\right) \text {. }
$$

In view of (2.7) and Lemma 2.1 we see that a.e. on $\partial E$,

$$
\begin{array}{r}
\varrho(\lambda)\left(\prod_{k=2}^{n} p_{j_{1}, \ldots, j_{k-1}}\left(J_{j_{1}, \ldots, j_{k-1}}\left(\varphi^{*}(\lambda)\right)\right)^{p_{j_{1}, \ldots, j_{k-1}}-1}\right)\left|\varphi_{j_{1}, \ldots, j_{n}}^{*}(\lambda)\right|^{2} \\
=\left|h_{j_{1}, \ldots, j_{n}}^{*}(\lambda) \varphi_{j_{1}, \ldots, j_{n}}^{*}(\lambda)\right|=r_{j_{1}, \ldots, j_{n}}\left|1-\bar{\alpha}_{j_{1}, \ldots, j_{n}} \lambda\right|^{2} .
\end{array}
$$

Summing now the left-hand side of $(2.14)$, for $\left(j_{1}, \ldots, j_{n-1}\right)$ fixed, with respect to $j_{n}$ from 1 to $m_{j_{1}, \ldots, j_{m_{j_{1}}, \ldots, j_{n-1}}}$ we get a.e. on $\partial E$ (we also 
use $(2.8))$

$$
\begin{aligned}
\varrho(\lambda)\left(\prod_{k=2}^{n-1} p_{j_{1}, \ldots, j_{k-1}}\left(J_{j_{1}, \ldots, j_{k-1}}\left(\varphi^{*}(\lambda)\right)\right)^{p_{j_{1}, \ldots, j_{k-1}}-1}\right) \\
\quad \times p_{j_{1}, \ldots, j_{n-1}}\left(\sum_{j_{n}=1}^{m_{j_{1}, \ldots, j_{n-1}}}\left|\varphi_{j_{1}, \ldots, j_{n}}^{*}(\lambda)\right|^{2}\right)^{p_{j_{1}, \ldots, j_{n-1}}} \\
=\varrho(\lambda) \sum_{j_{n}=1}^{m_{j_{1}, \ldots, j_{n-1}}}\left(\left(\prod_{k=2}^{n} p_{j_{1}, \ldots, j_{k-1}}\right.\right. \\
\left.\left.\quad \times\left(J_{j_{1}, \ldots, j_{k-1}}\left(\varphi^{*}(\lambda)\right)\right)^{p_{j_{1}, \ldots, j_{k-1}}-1}\right)\left|\varphi_{j_{1}, \ldots, j_{n}}^{*}(\lambda)\right|^{2}\right) \\
=\left|\sum_{j_{n}=1}^{m_{j_{1}, \ldots, j_{n-1}}} h_{j_{1}, \ldots, j_{n}}^{*}(\lambda) \varphi_{j_{1}, \ldots, j_{n}}^{*}(\lambda)\right| \\
=r_{j_{1}, \ldots, j_{n-1}}\left|1-\bar{\alpha}_{j_{1}, \ldots, j_{n-1}} \lambda\right|^{2} .
\end{aligned}
$$

Using again this procedure (this time summing the left-hand side of the previous equality w.r.t. $j_{n-1}$ with $\left(j_{1}, \ldots, j_{n-2}\right)$ fixed) we get

$$
\begin{aligned}
& \varrho(\lambda)\left(\prod_{k=2}^{n-2} p_{j_{1}, \ldots, j_{k-1}}\left(J_{j_{1}, \ldots, j_{k-1}}\left(\varphi^{*}(\lambda)\right)\right)^{p_{j_{1}, \ldots, j_{k-1}}-1}\right) \\
& \times p_{j_{1}, \ldots, j_{n-2}} \min _{j_{n-1}}\left\{p_{j_{1}, \ldots, j_{n-1}}\right\}\left(J_{j_{1}, \ldots, j_{n-2}}\left(\varphi^{*}(\lambda)\right)\right)^{p_{j_{1}, \ldots, j_{n-2}}} \\
& \leq \varrho(\lambda)\left(\prod_{k=2}^{n-2} p_{j_{1}, \ldots, j_{k-1}}\left(J_{j_{1}, \ldots, j_{k-1}}\left(\varphi^{*}(\lambda)\right)\right)^{p_{j_{1}, \ldots, j_{k-1}}-1}\right) \\
& \times p_{j_{1}, \ldots, j_{n-2}}\left(J_{j_{1}, \ldots, j_{n-2}}\left(\varphi^{*}(\lambda)\right)\right)^{p_{j_{1}, \ldots, j_{n-2}-1}} \\
& \times\left(\sum_{j_{n-1}=1}^{m_{j_{1}, \ldots, j_{n-2}}} p_{j_{1}, \ldots, j_{n-1}}\left(\sum_{j_{n}=1}^{m_{j_{1}, \ldots, j_{n-1}}}\left|\varphi_{j_{1}, \ldots, j_{n}}^{*}(\lambda)\right|^{2}\right)^{p_{j_{1}, \ldots, j_{n-1}}}\right) \\
& =\varrho(\lambda) \sum_{j_{n-1}=1}^{m_{j_{1}, \ldots, j_{n-2}}} \sum_{j_{n}=1}^{m_{j_{1}, \ldots, j_{n-1}}}\left(\left(\prod_{k=2}^{n} p_{j_{1}, \ldots, j_{k-1}}\right.\right. \\
& \left.\left.\times\left(J_{j_{1}, \ldots, j_{k-1}}\left(\varphi^{*}(\lambda)\right)\right)^{p_{j_{1}, \ldots, j_{k-1}}-1}\right)\left|\varphi_{j_{1}, \ldots, j_{n}}^{*}(\lambda)\right|^{2}\right) \\
& =\left|\sum_{j_{n-1}=1}^{m_{j_{1}, \ldots, j_{n-2}}} \sum_{j_{n}=1}^{m_{j_{1}, \ldots, j_{n-1}}} h_{j_{1}, \ldots, j_{n}}^{*}(\lambda) \varphi_{j_{1}, \ldots, j_{n}}^{*}(\lambda)\right| \\
& =r_{j_{1}, \ldots, j_{n-2}}\left|1-\bar{\alpha}_{j_{1}, \ldots, j_{n-2}}\right|^{2}
\end{aligned}
$$




$$
\begin{aligned}
\leq & \varrho(\lambda)\left(\prod_{k=2}^{n-2} p_{j_{1}, \ldots, j_{k-1}}\left(J_{j_{1}, \ldots, j_{k-1}}\left(\varphi^{*}(\lambda)\right)\right)^{p_{j_{1}, \ldots, j_{k-1}}-1}\right) \\
& \times p_{j_{1}, \ldots, j_{n-2}} \max _{j_{n-1}}\left\{p_{j_{1}, \ldots, j_{n-1}}\right\}\left(J_{j_{1}, \ldots, j_{n-2}}\left(\varphi^{*}(\lambda)\right)\right)^{p_{j_{1}, \ldots, j_{n-2}}} .
\end{aligned}
$$

And, generally,

$$
\begin{aligned}
(2.15) \varrho(\lambda)( & \left.\prod_{k=2}^{l} p_{j_{1}, \ldots, j_{k-1}}\left(J_{j_{1}, \ldots, j_{k-1}}\left(\varphi^{*}(\lambda)\right)\right)^{p_{j_{1}, \ldots, j_{k-1}}-1}\right) \\
& \times p_{j_{1}, \ldots, j_{l}} \min _{j_{n}, \ldots, j_{l+1}}\left\{p_{j_{1}, \ldots, j_{n}} \ldots p_{j_{1}, \ldots, j_{l+1}}\right\}\left(J_{j_{1}, \ldots, j_{l}}\left(\varphi^{*}(\lambda)\right)\right)^{p_{j_{1}, \ldots, j_{l}}} \\
\leq & r_{j_{1}, \ldots, j_{l}}\left|1-\bar{\alpha}_{j_{1}, \ldots, j_{l}} \lambda\right|^{2} \\
\leq & \varrho(\lambda)\left(\prod_{k=2}^{l} p_{j_{1}, \ldots, j_{k-1}}\left(J_{j_{1}, \ldots, j_{k-1}}\left(\varphi^{*}(\lambda)\right)\right)^{p_{j_{1}, \ldots, j_{k-1}}-1}\right) \\
& \times p_{j_{1}, \ldots, j_{l}} \max _{j_{n}, \ldots, j_{l+1}}\left\{p_{j_{1}, \ldots, j_{n}} \ldots p_{j_{1}, \ldots, j_{l+1}}\right\}\left(J_{j_{1}, \ldots, j_{l}}\left(\varphi^{*}(\lambda)\right)\right)^{p_{j_{1}, \ldots, j_{l}},},
\end{aligned}
$$

for $l=2, \ldots, n$ and moreover,

$$
\begin{aligned}
& p_{j_{1}} \varrho(\lambda) \min _{j_{n}, \ldots, j_{2}}\left\{p_{j_{1}, \ldots, j_{n}} \ldots p_{j_{1}, j_{2}}\right\}\left(J_{j_{1}}\left(\varphi^{*}(\lambda)\right)\right)^{p_{j_{1}}} \\
& \leq r_{j_{1}}\left|1-\bar{\alpha}_{j_{1}} \lambda\right|^{2} \leq p_{j_{1}} \varrho(\lambda) \max _{j_{n}, \ldots, j_{2}}\left\{p_{j_{1}, \ldots, j_{n}} \ldots p_{j_{1}, j_{2}}\right\}\left(J_{j_{1}}\left(\varphi^{*}(\lambda)\right)\right)^{p_{j_{1}}}
\end{aligned}
$$

and

$$
\begin{aligned}
& \varrho(\lambda) \min _{j_{n}, \ldots, j_{1}}\left\{p_{j_{1}, \ldots, j_{n}} \ldots p_{j_{1}}\right\} \\
& \quad=\varrho(\lambda) \min _{j_{n}, \ldots, j_{1}}\left\{p_{j_{1}, \ldots, j_{n}} \ldots p_{j_{1}}\right\}\left(J_{0}\left(\varphi^{*}(\lambda)\right)\right) \\
& \quad \leq r_{0}\left|1-\bar{\alpha}_{0} \lambda\right|^{2} \leq \varrho(\lambda) \max _{j_{n}, \ldots, j_{1}}\left\{p_{j_{1}, \ldots, j_{n}} \ldots p_{j_{1}}\right\}\left(J_{0}\left(\varphi^{*}(\lambda)\right)\right) \\
& \quad=\varrho(\lambda) \max _{j_{n}, \ldots, j_{1}}\left\{p_{j_{1}, \ldots, j_{n}} \ldots p_{j_{1}}\right\} .
\end{aligned}
$$

Set

$$
R_{j_{1}, \ldots, j_{l-1}}(\lambda):=\varrho(\lambda) \prod_{k=2}^{l} p_{j_{1}, \ldots, j_{k-1}}\left(J_{j_{1}, \ldots, j_{k-1}}\left(\varphi^{*}(\lambda)\right)\right)^{p_{j_{1}, \ldots, j_{k-1}-1}}
$$

for $\lambda \in \partial E$ a.e., $l=2, \ldots, n$, and additionally $R_{0}(\lambda):=\varrho(\lambda)$. Then it is clear that

(2.18) $\quad R_{j_{1}, \ldots, j_{l}}(\lambda)=R_{j_{1}, \ldots, j_{l-1}}(\lambda) p_{j_{1}, \ldots, j_{l}}\left(J_{j_{1}, \ldots, j_{l}}\left(\varphi^{*}(\lambda)\right)\right)^{p_{j_{1}, \ldots, j_{l}}-1}$ for $l=1, \ldots, n-1$. From (2.15) and (2.16) we get

(2.19) $\quad R_{j_{1}, \ldots, j_{l-1}}(\lambda) p_{j_{1}, \ldots, j_{l}} \min _{j_{n}, \ldots, j_{l+1}}\left\{p_{j_{1}, \ldots, j_{n}} \ldots p_{j_{1}, \ldots, j_{l+1}}\right\}$

$$
\times\left(J_{j_{1}, \ldots, j_{l}}\left(\varphi^{*}(\lambda)\right)\right)^{p_{j_{1}}, \ldots, j_{l}}
$$




$$
\begin{aligned}
\leq & r_{j_{1}, \ldots, j_{l}}\left|1-\bar{\alpha}_{j_{1}, \ldots, j_{l}} \lambda\right|^{2} \\
\leq & R_{j_{1}, \ldots, j_{l-1}}(\lambda) p_{j_{1}, \ldots, j_{l}} \\
& \quad \max _{j_{n}, \ldots, j_{l+1}}\left\{p_{j_{1}, \ldots, j_{n}} \ldots p_{j_{1}, \ldots, j_{l+1}}\right\}\left(J_{j_{1}, \ldots, j_{l}}\left(\varphi^{*}(\lambda)\right)\right)^{p_{j_{1}, \ldots, j_{l}}}
\end{aligned}
$$

so in other words,

$$
\begin{aligned}
& \frac{r_{j_{1}, \ldots, j_{l}}\left|1-\bar{\alpha}_{j_{1}, \ldots, j_{l}} \lambda\right|^{2}}{R_{j_{1}, \ldots, j_{l-1}}(\lambda) p_{j_{1}, \ldots, j_{l}}} \cdot \frac{1}{\mathcal{M}_{j_{1}, \ldots, j_{l}}} \\
& \quad \leq J_{j_{1}, \ldots, j_{l}}\left(\varphi^{*}(\lambda)\right)^{p_{j_{1}, \ldots, j_{l}}} \leq \frac{r_{j_{1}, \ldots, j_{l}}\left|1-\bar{\alpha}_{j_{1}, \ldots, j_{l}} \lambda\right|^{2}}{R_{j_{1}, \ldots, j_{l-1}}(\lambda) p_{j_{1}, \ldots, j_{l}}} \cdot \frac{1}{\mathcal{N}_{j_{1}, \ldots, j_{l}}},
\end{aligned}
$$

for $l=1, \ldots, n-1$, where $\mathcal{M}_{j_{1}, \ldots, j_{l}}$ (respectively $\mathcal{N}_{j_{1}, \ldots, j_{l}}$ ) is the maximum (respectively minimum) from the earlier formula; $\mathcal{M}_{0}$ (respectively $\mathcal{N}_{0}$ ) equals max (respectively min) with respect to $j_{1}, \ldots, j_{n}$ and $\mathcal{M}_{j_{1}, \ldots, j_{n}}:=$ $\mathcal{N}_{j_{1}, \ldots, j_{n}}:=1$.

From (2.14) and (2.18) we get, a.e. on $\partial E$,

$$
\begin{aligned}
&\left|\varphi_{j_{1}, \ldots, j_{n}}^{*}(\lambda)\right|^{2}=\frac{r_{j_{1}, \ldots, j_{n}}\left|1-\bar{\alpha}_{j_{1}, \ldots, j_{n}} \lambda\right|^{2}}{R_{j_{1}, \ldots, j_{n-1}}(\lambda)} \\
&=\frac{r_{j_{1}, \ldots, j_{n}}}{\tilde{\mathcal{M}}_{j_{1}, \ldots, j_{n} p_{j_{1}, \ldots, j_{n}}}}\left|1-\bar{\alpha}_{j_{1}, \ldots, j_{n}} \lambda\right|^{2} \\
& R_{j_{1}, \ldots, j_{n-2}}(\lambda) p_{j_{1}, \ldots, j_{n-1}}\left(J_{j_{1}, \ldots, j_{n-1}}\left(\varphi^{*}(\lambda)\right)\right)^{p_{j_{1}, \ldots, j_{n-1}-1}}
\end{aligned}
$$

where we put $\widetilde{\mathcal{M}}_{j_{1}, \ldots, j_{n}}:=1$; in view of (2.20) the last expression is at least

$$
\begin{aligned}
& \frac{\frac{r_{j_{1}, \ldots, j_{n}}}{\tilde{\mathcal{M}}_{j_{1}, \ldots, j_{n} p_{j_{1}, \ldots, j_{n}}}}\left|1-\bar{\alpha}_{j_{1}, \ldots, j_{n}} \lambda\right|^{2}}{R_{j_{1}, \ldots, j_{n-2}}(\lambda) p_{j_{1}, \ldots, j_{n-1}}} \\
& \times \frac{\left(\widetilde{\mathcal{M}}_{j_{1}, \ldots, j_{n-1}} p_{j_{1}, \ldots, j_{n-1}} R_{j_{1}, \ldots, j_{n-2}}(\lambda)\right)^{1-\frac{1}{p_{j_{1}, \ldots, j_{n-1}}}}}{\left(r_{j_{1}, \ldots, j_{n-1}}\left|1-\bar{\alpha}_{j_{1}, \ldots, j_{n-1}} \lambda\right|^{2}\right)^{1-\frac{1}{p_{j_{1}, \ldots, j_{n-1}}}}},
\end{aligned}
$$

where we put

$$
\widetilde{\mathcal{M}}_{j_{1}, \ldots, j_{k}}:= \begin{cases}\mathcal{N}_{j_{1}, \ldots, j_{k}} & \text { if } p_{j_{1}, \ldots, j_{k}} \geq 1 \\ \mathcal{M}_{j_{1}, \ldots, j_{k}} & \text { if } p_{j_{1}, \ldots, j_{k}}<1\end{cases}
$$

We define $\widetilde{\mathcal{N}}_{j_{1}, \ldots, j_{k}}$ by the same formula, but with the conditions interchanged (so it equals $\mathcal{N}_{j_{1}, \ldots, j_{k}}$ if $p_{j_{1}, \ldots, j_{k}}<1$ ). In other words, we have

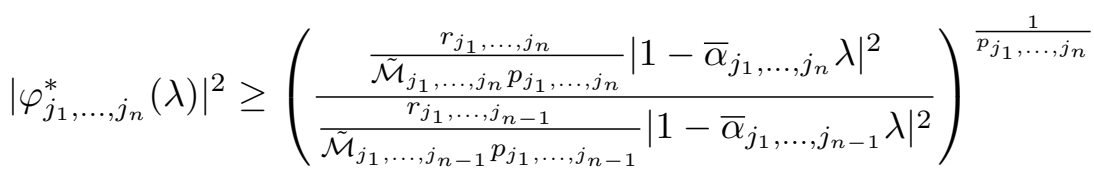




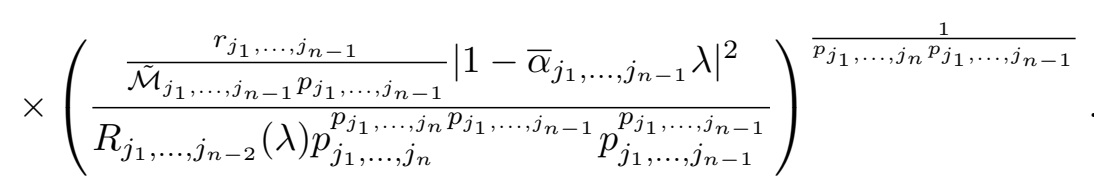

Repeating this procedure, in view of (2.18) and (2.20) we get

$$
\begin{aligned}
& \left|\varphi_{j_{1}, \ldots, j_{n}}^{*}(\lambda)\right|^{2}
\end{aligned}
$$

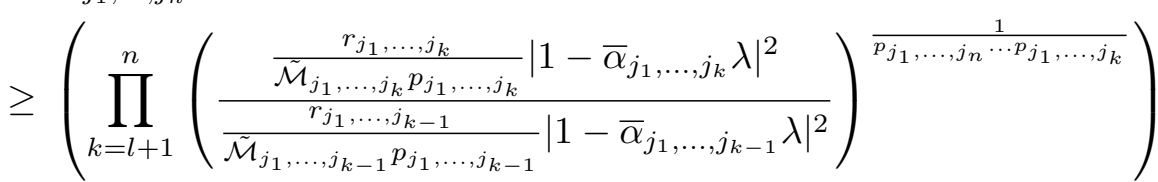

$$
\begin{aligned}
& \times\left(\frac{\frac{r_{j_{1}, \ldots, j_{l}}}{\tilde{\mathcal{M}}_{j_{1}, \ldots, j_{l}} p_{j_{1}, \ldots, j_{l}}}\left|1-\bar{\alpha}_{j_{1}, \ldots, j_{l}} \lambda\right|^{2}}{R_{j_{1}, \ldots, j_{l-1}}(\lambda) p_{j_{1}, \ldots, j_{n}}^{p_{j_{1}}, \ldots, j_{n} \ldots p_{j_{1}, \ldots, j_{l}} \ldots p_{j_{1}, \ldots, j_{l}}^{p_{j_{1}, \ldots, j_{l}}}}}\right)^{\frac{1}{p_{j_{1}, \ldots, j_{n} \cdots p_{j_{1}, \ldots, j_{l}}}}},
\end{aligned}
$$

for $l=1, \ldots, n-1$.

In view of (2.17) (remember that $R_{0}=\varrho$ ),

$$
\begin{aligned}
& \left|\varphi_{j_{1}, \ldots, j_{n}}^{*}(\lambda)\right|^{2}
\end{aligned}
$$

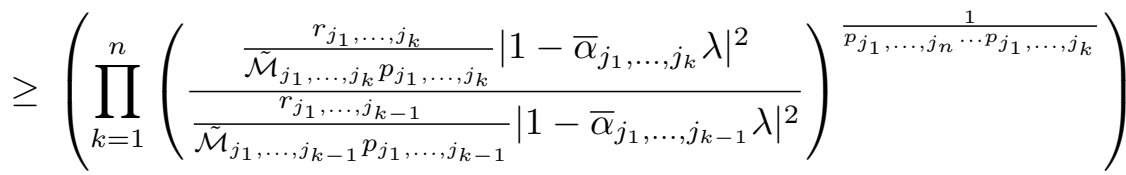

$$
\begin{aligned}
& \times\left(\frac{1}{p_{j_{1}, \ldots, j_{n}}^{p_{j_{1}, \ldots, j_{n}} \ldots p_{j_{1}}} \ldots p_{j_{1}}^{p_{j_{1}}}}\right)^{\frac{1}{p_{j_{1}, \ldots, j_{n} \cdots p_{j_{1}}}}} .
\end{aligned}
$$

We can also estimate $\varphi_{j_{1}, \ldots, j_{n}}$ from above analogously to (2.21) and (2.22); we get the same bound with $\widetilde{\mathcal{M}}_{j_{1}, \ldots, j_{k}}$ replaced by $\widetilde{\mathcal{N}}_{j_{1}, \ldots, j_{k}}$.

Note that if for some $l \in\{1, \ldots, n-1\}, p_{j_{1}, \ldots, j_{k}}$ is independent of the choice of $\left(j_{1}, \ldots, j_{k}\right)$ for any $k \in\{l+1, \ldots, n\}$, then in $(2.21)$ we have equality. More precisely, a.e. on $\partial E$,

$$
\begin{aligned}
& \left|\varphi_{j_{1}, \ldots, j_{n}}^{*}(\lambda)\right|^{2} \\
& =\left(\prod_{k=l+1}^{n}\left(\frac{r_{j_{1}, \ldots, j_{k}}\left|1-\bar{\alpha}_{j_{1}, \ldots, j_{k}} \lambda\right|^{2}}{r_{j_{1}, \ldots, j_{k-1}}\left|1-\bar{\alpha}_{j_{1}, \ldots, j_{k-1}} \lambda\right|^{2}}\right)^{\frac{1}{p_{j_{1}, \ldots, j_{n} \cdots p_{j_{1}, \ldots, j_{k}}}}}\right) \\
& \times\left(\frac{r_{j_{1}, \ldots, j_{l}}\left|1-\bar{\alpha}_{j_{1}, \ldots, j_{l}} \lambda\right|^{2}}{R_{j_{1}, \ldots, j_{l-1}}(\lambda) p_{j_{1}, \ldots, j_{n}} \ldots p_{j_{1}, \ldots, j_{l}}}\right)^{\frac{1}{p_{j_{1}, \ldots, j_{n}} \cdots p_{j_{1}, \ldots, j_{l}}}} .
\end{aligned}
$$

Note also that in view of (2.14) and (2.22), a.e. on $\partial E$,

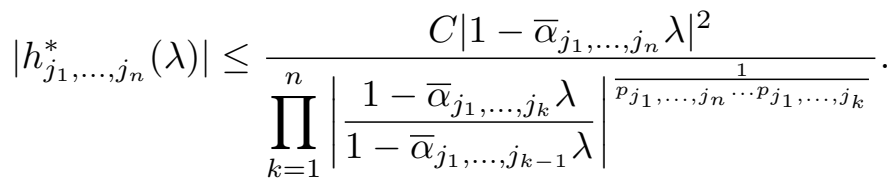


If $\alpha_{j_{1}, \ldots, j_{k}} \in E$ for all possible $\left(j_{1}, \ldots, j_{k}\right)$ (or $\left|\alpha_{0}\right|=1$ and then see (2.13)), then $h_{j_{1}, \ldots, j_{n}} \in H^{\infty}(E)$. But if $\left|\alpha_{j_{1}, \ldots, j_{k}}\right|=1$ for some $k$ and $\alpha_{j_{1}, \ldots, j_{k-1}} \in E$, then (see (2.12))

$$
h_{j_{1}, \ldots, j_{n}}(\lambda)\left(1-\bar{\alpha}_{j_{1}, \ldots, j_{k}} \lambda\right)^{\frac{1}{p_{j_{1}, \ldots, j_{n} \cdots p_{j_{1}}, \ldots, j_{k}}}-2} \in H^{\infty}(E) .
$$

In particular, $h_{j_{1}, \ldots, j_{n}} \in H^{\infty}(E)$ (see (1.3)).

Consider now the special case

$$
p_{j_{1}, \ldots, j_{l}}=\widetilde{q}_{l} \quad \text { for } l=1, \ldots, n .
$$

In this case we have a.e. on $\partial E$ (see (2.23) for $l=1$-remember that $R_{0} \widetilde{q}_{1} \ldots \widetilde{q}_{n}=\varrho \widetilde{q}_{1} \ldots \widetilde{q}_{n}=r_{0}\left|1-\bar{\alpha}_{0} \lambda\right|^{2}$ in view of $\left.(2.17)\right)$

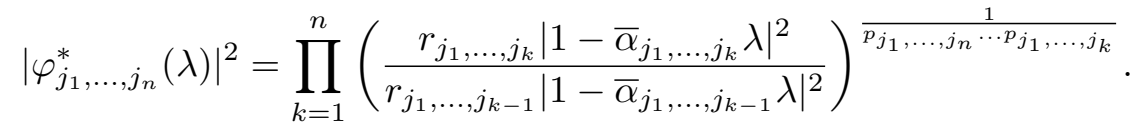

Since

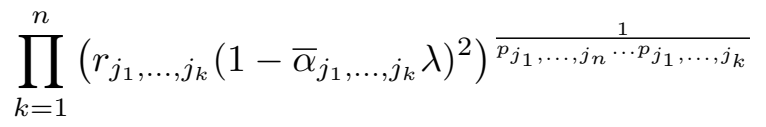

is an outer function, the decomposition theorem (see [Ga]) implies that for $\lambda \in E$,

$$
\begin{aligned}
& \varphi_{j_{1}, \ldots, j_{n}}(\lambda)=B_{j_{1}, \ldots, j_{n}}(\lambda) \\
& \quad \times\left(\prod_{k=1}^{n} a_{j_{1}, \ldots, j_{k}}\left(\frac{1-\bar{\alpha}_{j_{1}, \ldots, j_{k}} \lambda}{1-\bar{\alpha}_{j_{1}, \ldots, j_{k-1}} \lambda}\right)^{\frac{1}{p_{j_{1}, \ldots, j_{n}} \ldots p_{j_{1}, \ldots, j_{k}}}}\right) S_{j_{1}, \ldots, j_{n}}(\lambda),
\end{aligned}
$$

where

$$
\begin{aligned}
\left|a_{j_{1}, \ldots, j_{k}}\right| & =\left(\frac{r_{j_{1}, \ldots, j_{k}}}{r_{j_{1}, \ldots, j_{k-1}}}\right)^{1 / 2} \quad \text { for } k=1, \ldots, n, \\
B_{j_{1}, \ldots, j_{n}}(\lambda) & = \begin{cases}\frac{\lambda-\alpha_{j_{1}, \ldots, j_{n}}}{1-\bar{\alpha}_{j_{1}, \ldots, j_{n}} \lambda} & \text { if } s_{j_{1}, \ldots, j_{n}}=1, \\
1 & \text { if } s_{j_{1}, \ldots, j_{n}}=0,\end{cases} \\
S_{j_{1}, \ldots, j_{n}}(\lambda) & =\exp \left(-\int_{-\pi}^{\pi} \frac{e^{i \theta}+\lambda}{e^{i \theta}-\lambda} d \sigma_{j_{1}, \ldots, j_{n}}(\theta)\right),
\end{aligned}
$$

where $\sigma_{j_{1}, \ldots, j_{n}}$ is a singular nonnegative Borel measure.

It is sufficient to prove that $\sigma_{j_{1}, \ldots, j_{n}}=0$, because then, in view of (2.13), if $\left|\alpha_{0}\right|=1$, then $\alpha_{j_{1}, \ldots, j_{k}}=\alpha_{0}$ and $s_{j_{1}, \ldots, j_{n}}=0$ for all possible $\left(j_{1}, \ldots, j_{k}\right)$, which implies that $\varphi$ is constant-a contradiction; so $\alpha_{0} \in E$. Moreover, (2.9) and (2.10) yield (2.3) and (2.4). 
To prove that $\sigma_{j_{1}, \ldots, j_{n}}=0$ note that in view of $(2.25),(2.7)$ and the fact that $h_{j_{1}, \ldots, j_{n}} \in H^{\infty}(E)$ we get

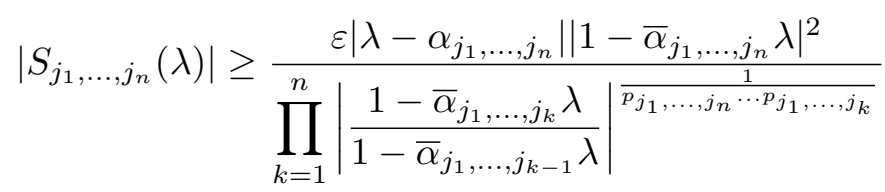

for $\lambda \in E$ and some $\varepsilon>0$. But

$$
S_{j_{1}, \ldots, j_{n}}^{*}(\lambda)=0 \quad \text { for } \sigma_{j_{1}, \ldots, j_{n}} \text {-almost all } \lambda \in \partial E .
$$

From these two conditions together with (2.12) we deduce, in view of the fact that the function

$$
E \ni \lambda \rightarrow|\lambda-1|^{\beta} \exp \left(b \frac{1-|\lambda|^{2}}{|\lambda-1|^{2}}\right), \quad \beta \in \mathbb{R}, b>0,
$$

is unbounded, that $\sigma_{j_{1}, \ldots, j_{n}}=0$.

Now consider the general case. We use induction. The previous case is the first inductive step. Assume that we have already proved the formulas for $p_{j_{1}, \ldots, j_{k}}=\widetilde{q}_{k}$ for $k=n, \ldots, l$.

Assume now that for $l<n$,

$$
p_{j_{1}, \ldots, j_{k}}=\widetilde{q}_{k} \quad \text { for } k=n, \ldots, l+1 .
$$

We may write (compare (2.23))

$$
\begin{aligned}
& \varphi_{j_{1}, \ldots, j_{n}}(\lambda) \\
& =B_{j_{1}, \ldots, j_{n}}(\lambda)
\end{aligned}
$$

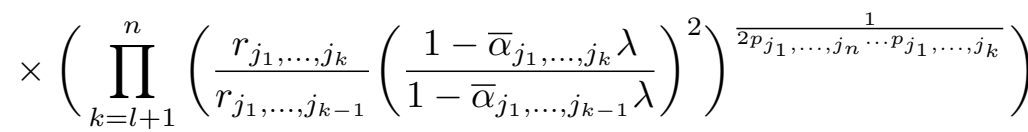

$$
\begin{aligned}
& \times \psi_{j_{1}, \ldots, j_{n}}(\lambda)
\end{aligned}
$$

for $\lambda \in E$, where $r_{j_{1}, \ldots, j_{k}}, \alpha_{j_{1}, \ldots, j_{k}}$ are as in (2.7) and (2.8) and $B_{j_{1}, \ldots, j_{n}}$ is the Blaschke product of $\varphi_{j_{1}, \ldots, j_{n}}$.

In view of $(2.23)$ it is clear that $\left|\psi_{j_{1}, \ldots, j_{n}}^{*}(\lambda)\right|$ is independent of the choice of $\left(j_{l+1}, \ldots, j_{n}\right)$ a.e. on $\partial E$ and $(2.20)$ implies additionally that

$$
\psi_{j_{1}, \ldots, j_{n}} \in H^{\infty}(E) .
$$

Define

$$
\begin{aligned}
\widetilde{p}_{j_{1}, \ldots, j_{k}} & :=p_{j_{1}, \ldots, j_{k}} \quad \text { for } k \neq l \\
\widetilde{p}_{j_{1}, \ldots, j_{l}} & :=\widetilde{q}_{l}:=\max _{j_{1}, \ldots, j_{l}}\left\{p_{j_{1}, \ldots, j_{l}}\right\}
\end{aligned}
$$


Let us also put

$$
\begin{aligned}
& \widetilde{\varphi}_{j_{1}, \ldots, j_{n}}(\lambda) \\
& :=B_{j_{1}, \ldots, j_{n}}(\lambda)
\end{aligned}
$$

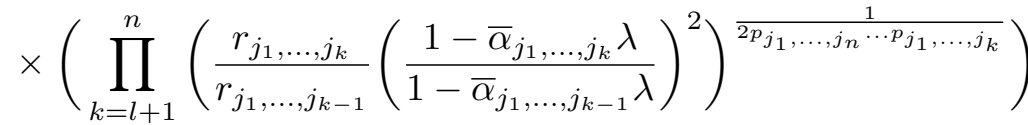

$$
\begin{aligned}
& \times \psi_{j_{1}, \ldots, j_{n}}(\lambda)^{p_{j_{1}, \ldots, j_{l}} / \tilde{q}_{l}}
\end{aligned}
$$

and

$$
\begin{aligned}
\widetilde{h}_{j_{1}, \ldots, j_{n}} & :=\frac{\widetilde{q}_{l}}{p_{j_{1}, \ldots, j_{l}}} h_{j_{1}, \ldots, j_{n}} \frac{\varphi_{j_{1}, \ldots, j_{n}}}{\widetilde{\varphi}_{j_{1}, \ldots, j_{n}}} \\
& =\frac{\widetilde{q}_{l}}{p_{j_{1}, \ldots, j_{l}}} h_{j_{1}, \ldots, j_{n}} \psi_{j_{1}, \ldots, j_{n}}^{1-p_{j_{1}, \ldots, j_{l}} / \tilde{q}_{l}},
\end{aligned}
$$

where $h_{j_{1}, \ldots, j_{n}}$ is chosen from Lemma 2.1 as before.

By $(2.27), \widetilde{h}_{j_{1}, \ldots, j_{n}} \in H^{1}(E)$ (even more, it is in $H^{\infty}(E)$ - see $(2.24)$ ).

In view of $(2.9),(2.10),(2.26)$ and the fact that $\left|\psi_{j_{1}, \ldots, j_{n}}^{*}(\lambda)\right|$ does not depend on $\left(j_{l+1}, \ldots, j_{n}\right)$, we have a.e. on $\partial E$,

$$
\begin{aligned}
& J_{j_{1}, \ldots, j_{n-1}}\left(\varphi^{*}(\lambda)\right)=\sum_{j_{n}=1}^{m_{j_{1}, \ldots, j_{n-1}}}\left|\varphi_{j_{1}, \ldots, j_{n}}^{*}(\lambda)\right|^{2} \\
& =\left|\psi_{j_{1}, \ldots, j_{n}}^{*}(\lambda)\right|^{2} \\
& \times\left(\sum _ { j _ { n } = 1 } ^ { m _ { j _ { 1 } } , \ldots , j _ { n - 1 } } \left(\prod _ { k = l + 1 } ^ { n } \left(\frac{r_{j_{1}, \ldots, j_{k}}}{r_{j_{1}, \ldots, j_{k-1}}}\right.\right.\right. \\
& \left.\left.\left.\times \frac{\left|1-\bar{\alpha}_{j_{1}, \ldots, j_{k}} \lambda\right|^{2}}{\left|1-\bar{\alpha}_{j_{1}, \ldots, j_{k-1}} \lambda\right|^{2}}\right)^{\frac{1}{p_{j_{1}, \ldots, j_{n-1} \cdots p_{j_{1}, \ldots, j_{k}}}}}\right)\right) \\
& =\left|\psi_{j_{1}, \ldots, j_{n}}^{*}(\lambda)\right|^{2}\left(\prod _ { k = l + 1 } ^ { n - 1 } \left(\frac{r_{j_{1}, \ldots, j_{k}}}{r_{j_{1}, \ldots, j_{k-1}}}\right.\right. \\
& \left.\left.\times \frac{\left|1-\bar{\alpha}_{j_{1}, \ldots, j_{k}} \lambda\right|^{2}}{\left|1-\bar{\alpha}_{j_{1}, \ldots, j_{k-1}} \lambda\right|^{2}}\right)^{\frac{1}{p_{j_{1}, \ldots, j_{n-1} \cdots p_{j_{1}, \ldots, j_{k}}}}}\right) \\
& \times \sum_{j_{n}=1}^{m_{j_{1}, \ldots, j_{n-1}}} \frac{r_{j_{1}, \ldots, j_{k}}}{r_{j_{1}, \ldots, j_{k-1}}} \cdot \frac{\left|1-\bar{\alpha}_{j_{1}, \ldots, j_{k}} \lambda\right|^{2}}{\left|1-\bar{\alpha}_{j_{1}, \ldots, j_{k-1}} \lambda\right|^{2}}
\end{aligned}
$$




$$
\begin{aligned}
& =\left|\psi_{j_{1}, \ldots, j_{n}}^{*}(\lambda)\right|^{2} \\
& \times\left(\prod_{k=l+1}^{n-1}\left(\frac{r_{j_{1}, \ldots, j_{k}}}{r_{j_{1}, \ldots, j_{k-1}}} \cdot \frac{\left|1-\bar{\alpha}_{j_{1}, \ldots, j_{k}} \lambda\right|^{2}}{\left|1-\bar{\alpha}_{j_{1}, \ldots, j_{k-1}} \lambda\right|^{2}}\right)^{\frac{1}{p_{j_{1}, \ldots, j_{n-1}} \ldots p_{j_{1}, \ldots, j_{k}}}}\right) .
\end{aligned}
$$

Moreover, if $n-1 \geq l+1$,

$$
\begin{aligned}
J_{j_{1}, \ldots, j_{n-2}} & \left(\varphi^{*}(\lambda)\right) \\
= & \sum_{j_{n-1}=1}^{m_{j_{1}, \ldots, j_{n-2}}} J_{j_{1}, \ldots, j_{n-1}}\left(\varphi^{*}(\lambda)\right)^{p_{j_{1}, \ldots, j_{n-1}}} \\
= & \left|\psi_{j_{1}, \ldots, j_{n}}^{*}(\lambda)\right|^{2 p_{j_{1}, \ldots, j_{n-1}}} \\
& \times \prod_{k=l+1}^{n-2}\left(\frac{r_{j_{1}, \ldots, j_{k}}}{r_{j_{1}, \ldots, j_{k-1}}} \cdot \frac{\left|1-\bar{\alpha}_{j_{1}, \ldots, j_{k}} \lambda\right|^{2}}{\left|1-\bar{\alpha}_{j_{1}, \ldots, j_{k-1}} \lambda\right|^{2}}\right)^{\frac{1}{p_{j_{1}, \ldots, j_{n-2}} \ldots p_{j_{1}, \ldots, j_{k}}}} .
\end{aligned}
$$

Generally, if $s+1 \geq l+1$, then

$$
\begin{aligned}
J_{j_{1}, \ldots, j_{s}}\left(\varphi^{*}(\lambda)\right)=\left|\psi_{j_{1}, \ldots, j_{n}}^{*}(\lambda)\right|^{2 p_{j_{1}}, \ldots, j_{n} \ldots p_{j_{1}}, \ldots, j_{s+1}} & \\
\times & \times \prod_{k=l+1}^{s}\left(\frac{r_{j_{1}, \ldots, j_{k}}}{r_{j_{1}, \ldots, j_{k-1}}} \cdot \frac{\left|1-\bar{\alpha}_{j_{1}, \ldots, j_{k}} \lambda\right|^{2}}{\left|1-\bar{\alpha}_{j_{1}, \ldots, j_{k-1}} \lambda\right|^{2}}\right)^{\frac{1}{p_{j_{1}, \ldots, j_{s} \cdots p_{j_{1}}, \ldots, j_{k}}}}
\end{aligned}
$$

In particular, we have (for $s=l$ )

$$
J_{j_{1}, \ldots, j_{l}}\left(\varphi^{*}(\lambda)\right)=\left|\psi_{j_{1}, \ldots, j_{n}}^{*}(\lambda)\right|^{2 p_{j_{1}, \ldots, j_{n}} \ldots p_{j_{1}, \ldots, j_{l+1}}} .
$$

Note that for $s<l$,

$$
\begin{aligned}
& J_{j_{1}, \ldots, j_{s}}\left(\varphi^{*}(\lambda)\right) \\
& =\sum_{j_{s}=1}^{m_{j_{1}}, \ldots, j_{s-1}}\left(\ldots\left(\sum_{j_{l}=1}^{m_{j_{1}, \ldots, j_{l-1}}} J_{j_{1}, \ldots, j_{l}}^{p_{j_{1}}, \ldots, j_{l}}\left(\varphi^{*}(\lambda)\right)\right)^{p_{j_{1}, \ldots, j_{l}-1}} \ldots\right)^{p_{j_{1}, \ldots, j_{s}}} .
\end{aligned}
$$

But

$$
\widetilde{J}_{j_{1}, \ldots, j_{l}}\left(\widetilde{\varphi}^{*}(\lambda)\right)^{\tilde{q}_{l}}=\left|\psi_{j_{1}, \ldots, j_{n}}^{*}(\lambda)^{p_{j_{1}}, \ldots, j_{l} / \tilde{q}_{l}}\right|^{2 \tilde{q}_{n} \ldots . \tilde{q}_{l}}=J_{j_{1}, \ldots, j_{l}}\left(\varphi^{*}(\lambda)\right)^{p_{j_{1}, \ldots, j_{l}}},
$$

where $\widetilde{J}$ is an analogue to $J$ with $\widetilde{p}_{l}$ in place of $p_{j_{1}, \ldots, j_{l}}$ (the formulas analogous to (2.30) and (2.31) with $\psi_{j_{1}, \ldots, j_{n}}^{p_{j_{1}, \ldots, j_{l}} / \tilde{q}_{l}}$ in place of $\psi_{j_{1}, \ldots, j_{n}}$ also hold for $\widetilde{J}$ and $\widetilde{\varphi})$. This implies that

$$
\widetilde{J}_{j_{1}, \ldots, j_{s}}\left(\widetilde{\varphi}^{*}(\lambda)\right)=J_{j_{1}, \ldots, j_{s}}\left(\varphi^{*}(\lambda)\right)
$$

for any $s<l$. In particular,

$$
\widetilde{J}_{0}\left(\widetilde{\varphi}^{*}(\lambda)\right)=1 \quad \text { a.e. on } \partial E .
$$

To prove that $\widetilde{\varphi}$ is a geodesic in an ellipsoid with $\widetilde{q_{l}}$ in place of $p_{j_{1}, \ldots, j_{l}}$ (note that in view of the definition of $\widetilde{q}_{l}$ the new pseudoellipsoid satisfies 
(1.3)) we verify that

$$
\begin{aligned}
& \frac{1}{\lambda} \widetilde{\varphi}_{j_{1}, \ldots, j_{n}}^{*}(\lambda) \widetilde{h}_{j_{1}, \ldots, j_{n}}^{*}(\lambda) \\
& \quad=\varrho(\lambda)\left(\prod_{k=2}^{n} p_{j_{1}, \ldots, j_{k-1}}\left(\widetilde{J}_{j_{1}, \ldots, j_{k-1}}\left(\widetilde{\varphi}^{*}(\lambda)\right)\right)^{p_{j_{1}, \ldots, j_{k-1}}-1}\right)\left|\tilde{\varphi}_{j_{1}, \ldots, j_{n}}^{*}(\lambda)\right|^{2}
\end{aligned}
$$

a.e. on $\partial E$. By (2.29) we have

$$
\frac{1}{\lambda} \widetilde{\varphi}_{j_{1}, \ldots, j_{n}}^{*}(\lambda) \widetilde{h}_{j_{1}, \ldots, j_{n}}^{*}(\lambda)=\frac{\widetilde{q}_{l}}{p_{j_{1}, \ldots, j_{l}}} \frac{1}{\lambda} \varphi_{j_{1}, \ldots, j_{n}}^{*}(\lambda) h_{j_{1}, \ldots, j_{n}}^{*}(\lambda),
$$

which, in view of Lemma 2.1 applied to $\varphi$, is equal to

$$
\begin{aligned}
(2.33) \frac{\widetilde{q}_{l}}{p_{j_{1}, \ldots, j_{l}}} & \varrho(\lambda) \\
& \times\left(\prod_{k=2}^{n} p_{j_{1}, \ldots, j_{k-1}}\left(J_{j_{1}, \ldots, j_{k-1}}\left(\varphi^{*}(\lambda)\right)\right)^{p_{j_{1}, \ldots, j_{k-1}}-1}\right)\left|\varphi_{j_{1}, \ldots, j_{n}}^{*}(\lambda)\right|^{2} .
\end{aligned}
$$

From (2.30) we get

$$
\begin{aligned}
& \frac{\widetilde{q}_{l}}{p_{j_{1}, \ldots, j_{l}}} \varrho(\lambda)\left(\prod_{k=l+1}^{n} p_{j_{1}, \ldots, j_{k-1}}\left(J_{j_{1}, \ldots, j_{k-1}}\left(\varphi^{*}(\lambda)\right)\right)^{p_{j_{1}, \ldots, j_{k-1}}-1}\right) \\
& \times\left|\varphi_{j_{1}, \ldots, j_{n}}^{*}(\lambda)\right|^{2} \\
& =\varrho(\lambda)\left(\prod _ { k = l + 1 } ^ { n } \left(\widetilde{q}_{k-1}\left|\psi_{j_{1}, \ldots, j_{n}}^{*}(\lambda)\right|^{2 \tilde{q}_{n} \ldots \tilde{q}_{k}\left(p_{j_{1}, \ldots, j_{k-1}}-1\right)}\right.\right. \\
& \left.\left.\times \prod_{t=l+1}^{k-1}\left(\frac{r_{j_{1}, \ldots, j_{t}}}{r_{j_{1}, \ldots, j_{t-1}}} \cdot \frac{\left|1-\bar{\alpha}_{j_{1}, \ldots, j_{t}} \lambda\right|^{2}}{\left|1-\bar{\alpha}_{j_{1}, \ldots, j_{t-1}} \lambda\right|^{2}}\right)^{\frac{p_{p_{j_{1}}, \ldots, j_{k-1} \cdots j_{k-1}-1} \cdots p_{j_{1}}, \ldots, j_{t}}{p_{j_{1}}}}\right)\right) \\
& \times\left|\psi_{j_{1}, \ldots, j_{n}}^{*}(\lambda)\right|^{2} \\
& \times \prod_{k=l+1}^{n}\left(\frac{r_{j_{1}, \ldots, j_{k}}}{r_{j_{1}, \ldots, j_{k-1}}} \cdot \frac{\left|1-\bar{\alpha}_{j_{1}, \ldots, j_{k}} \lambda\right|^{2}}{\left|1-\bar{\alpha}_{j_{1}, \ldots, j_{k-1}} \lambda\right|^{2}}\right)^{\frac{1}{p_{j_{1}, \ldots, j_{n} \cdots p_{j_{1}}, \ldots, j_{k}}}} \\
& =\varrho(\lambda)\left(\prod _ { k = l + 1 } ^ { n } \left(\widetilde{q}_{k-1}\left|\psi_{j_{1}, \ldots, j_{n}}^{*}(\lambda)^{p_{j_{1}, \ldots, j_{l}} / \tilde{q}_{l}}\right|^{2 \tilde{q}_{n} \ldots \tilde{q}_{k}\left(\tilde{q}_{k-1}-1\right)}\right.\right. \\
& \left.\left.\times \prod_{t=l+1}^{k-1}\left(\frac{r_{j_{1}, \ldots, j_{t}}}{r_{j_{1}, \ldots, j_{t-1}}} \cdot \frac{\left|1-\bar{\alpha}_{j_{1}, \ldots, j_{t}} \lambda\right|^{2}}{\left|1-\bar{\alpha}_{j_{1}, \ldots, j_{t-1}} \lambda\right|^{2}}\right)^{\frac{p_{j_{1}, \ldots, j_{k-1}-1}}{\bar{p}_{j_{1}, \ldots, j_{k-1} \cdots p_{j_{1}}, \ldots, j_{t}}}}\right)\right) \\
& \times\left|\psi_{j_{1}, \ldots, j_{n}}^{*}(\lambda)^{p_{j_{1}, \ldots, j_{l}} / \tilde{q}_{l}}\right|^{2}
\end{aligned}
$$




$$
\begin{aligned}
& \times \prod_{k=l+1}^{n}\left(\frac{r_{j_{1}, \ldots, j_{k}}}{r_{j_{1}, \ldots, j_{k-1}}} \cdot \frac{\left|1-\bar{\alpha}_{j_{1}, \ldots, j_{k}} \lambda\right|^{2}}{\left|1-\bar{\alpha}_{j_{1}, \ldots, j_{k-1}} \lambda\right|^{2}}\right)^{\frac{1}{p_{j_{1}, \ldots, j_{n} \ldots p_{j_{1}}, \ldots, j_{k}}}} \\
= & \varrho(\lambda)\left(\prod_{k=l+1}^{n} \widetilde{p}_{j_{1}, \ldots, j_{k-1}}\left(\widetilde{J}_{j_{1}, \ldots, j_{k-1}}\left(\widetilde{\varphi}^{*}(\lambda)\right)\right)^{\tilde{p}_{j_{1}, \ldots, j_{k-1}}-1}\right)\left|\widetilde{\varphi}_{j_{1}, \ldots, j_{n}}^{*}(\lambda)\right|^{2} .
\end{aligned}
$$

The last but one equality is a consequence of the fact that

$$
\begin{aligned}
& \sum_{k=l+1}^{n} 2 \widetilde{q}_{n} \ldots \widetilde{q}_{k}\left(p_{j_{1}, \ldots, j_{k-1}}-1\right)+2 \\
&=\sum_{k=l+1}^{n} 2 \widetilde{q}_{n} \ldots \widetilde{q}_{k}\left(\widetilde{q}_{k-1}-1\right) \frac{p_{j_{1}, \ldots, j_{l}}}{\widetilde{q}_{l}}+2 \frac{p_{j_{1}, \ldots, j_{l}}}{\widetilde{q}_{l}} .
\end{aligned}
$$

Together with (2.32) and (2.33), this completes the proof of the fact that $\widetilde{\varphi}$ is a geodesic in a suitable ellipsoid. In view of the inductive assumption applied to $\widetilde{\varphi}$ and because of the form of $\varphi$ and $\widetilde{\varphi}, h$ and $\widetilde{h}$ the proof of the inductive step is complete. For $l=n-1$ we obtain the assertion of Theorem 2.2.

Remark 2.3. If $\varphi$ is a geodesic and $\varphi_{j_{1}, \ldots, j_{n}} \equiv 0$ for some $\left(j_{1}, \ldots, j_{n}\right)$, then the mapping $\widetilde{\varphi}: E \rightarrow \mathbb{C}^{N-1}$ all of whose components but $\left(j_{1}, \ldots, j_{n}\right)$ are equal to the components of $\varphi$ and the $\left(j_{1}, \ldots, j_{n}\right)$ component is omitted, is a geodesic in a suitable pseudoellipsoid in $\mathbb{C}^{N-1}$, which is naturally convex if $\mathcal{E}$ is convex.

Remark 2.4. In case $n=2, m_{j}=1$ for $j=1, \ldots, m_{0}$, Theorem 2.2 has been proved in [JPZ] (see also [JP]). Moreover, those works show the uniqueness of the geodesics, up to automorphisms of $E$; for strictly convex bounded domains this uniqueness is a general property (see $[D]$ ) and the complex ellipsoids are convex and not strictly convex if $p_{j} \geq 1 / 2$ for $j=$ $1, \ldots, m_{0}$ and $\#\left\{j: p_{j}=1 / 2\right\}>1$.

Remark 2.5. The formulas of Theorem 2.2 show that the geodesics extend continuously to the boundary. In many cases one can get more information about the regularity of the extension. Moreover, it is a relatively rare phenomenon that the extensions of all geodesics are holomorphic on some neighbourhoods of $\bar{E}$. This is so, for instance, when all the products in (1.3) are 1 or $1 / 2$ (see also the proof of Proposition 3.2).

Remark 2.6. From Theorem 2.2 it is clear that if $\left|\alpha_{\jmath_{1}, \ldots, j_{k}}\right|=1$ for some $k \in\{1, \ldots, n-1\}$, then $\alpha_{\jmath_{1}, \ldots, \jmath_{k}, j_{k+1}, \ldots, j_{l}}=\alpha_{\jmath_{1}, \ldots, j_{k}}$ for $l>k$ (the $\alpha$ 's are as in the formulas for complex geodesics in $\mathcal{E}$ in Theorem 2.2). 


\section{Automorphisms of convex generalized pseudoellipsoids}

Lemma 3.1. Assume that $\varphi$ and $\mathcal{E}$ are as in Theorem 2.2. Fix $\left(j_{1}, \ldots, \jmath_{k}\right)$ with $k \in\{1, \ldots, n-1\}$. If $\alpha_{\jmath_{1}, \ldots, j_{k}, j_{k+1}}=1$ for $j_{k+1}=1, \ldots, m_{\jmath_{1}, \ldots, j_{k}}$, then $\alpha_{\jmath_{1}, \ldots, j_{k}}=1$.

Proof. From Theorem 2.2 we get

$$
\alpha_{\jmath_{1}, \ldots, \jmath_{k}}=\sum_{j_{k+1}=1}^{m_{\jmath_{1}, \ldots, \jmath_{k}}}\left|a_{\jmath_{1}, \ldots, \jmath_{k}, j_{k+1}}\right|^{2} \alpha_{\jmath_{1}, \ldots, \jmath_{k}, j_{k+1}}=\sum_{j_{k+1}=1}^{m_{\jmath_{1}, \ldots, \jmath_{k}}}\left|a_{\jmath_{1}, \ldots, \jmath_{k}, j_{k+1}}\right|^{2} .
$$

Moreover,

$$
\begin{aligned}
1+\alpha_{\jmath_{1}, \ldots, \jmath_{k}}^{2} & =1+\left|\alpha_{\jmath_{1}, \ldots, \jmath_{k}}\right|^{2}=\sum_{j_{k+1}=1}^{m_{\jmath_{1}, \ldots, \rho_{k}}}\left|a_{\jmath_{1}, \ldots, \jmath_{k}, j_{k+1}}\right|^{2}\left(1+\left|\alpha_{\jmath_{1}, \ldots, \jmath_{k}, j_{k+1}}\right|^{2}\right) \\
& =2 \sum_{j_{k+1}=1}^{m_{\jmath_{1}, \ldots, \jmath_{k}}}\left|a_{\jmath_{1}, \ldots, \jmath_{k}, j_{k+1}}\right|^{2}=2 \alpha_{\jmath_{1}, \ldots, \jmath_{k}} .
\end{aligned}
$$

The last equality completes the proof.

Let $\mathcal{E}$ be a generalized pseudoellipsoid as before, but, additionally, we assume that we may present it in the following way:

$$
\begin{aligned}
\mathcal{E}:=\left\{\sum_{j_{1}=1}^{m_{0}}\left(\ldots\left(\sum_{j_{n}=1}^{m_{j_{1}}, \ldots, j_{n-1}}\left|z_{j_{1}, \ldots, j_{n}}\right|^{2}\right)^{p_{j_{1}}, \ldots, j_{n-1}} \ldots\right)^{p_{j_{1}}}\right. \\
\left.+\left|z_{m_{0}+1}\right|^{2}+\ldots+\left|z_{m_{0}+r}\right|^{2}<1\right\},
\end{aligned}
$$

where $r$ is largest possible. We may assume without loss of generality that if $r=0$, then $m_{0}>1$.

Assume that $\mathcal{E} \subset \mathbb{C}^{N}$, where $N=N_{1}+r$.

Proposition 3.2. Let $\Phi: D \rightarrow \mathcal{E}$ be a biholomorphic mapping, where $D$ is a bounded convex complete Reinhardt domain. Assume that the coefficients $p$ in the definition of $\mathcal{E}$ satisfy

$$
\begin{aligned}
p_{j_{1}, \ldots, j_{n}} \ldots p_{j_{1}, \ldots, j_{k}} & >1 / 2 \\
& \text { for any possible }\left(j_{1}, \ldots, j_{n}\right) \text { and } k=1, \ldots, n .
\end{aligned}
$$

If $\Phi(0)=b$, where

$$
b_{m_{0}+1}=\ldots=b_{m_{0}+r}=0,
$$

then $b=0$.

P r o of. We restrict our attention to the case $\operatorname{dim} \mathcal{E}>1$, keeping in mind that $\Phi$ extends to a biholomorphism between some neighbourhoods of $\bar{D}$ and $\overline{\mathcal{E}}$ (see $[\mathrm{B}]$, also $[\mathrm{JP}]$ ). 
Suppose that $b \neq 0$. Then $N_{1}>0$. In other words, there is $\left(\jmath_{1}, \ldots, \jmath_{n}\right)$ such that

$$
b_{\jmath_{1}, \ldots, \jmath_{n}} \neq 0 .
$$

Changing $p$ 's if necessary so that they define the same pseudoellipsoid $\mathcal{E}$ and satisfy (3.1) we may assume that there are $k \in\{1, \ldots, n-1\}$ and $\left(j_{1}, \ldots, j_{n}\right)$ such that

$$
\begin{aligned}
& p_{\jmath_{1}, \ldots, \jmath_{n}} \ldots p_{\jmath_{1}, \ldots, \jmath_{k}} \neq 1 \text { and if } k \geq 2, \text { then } p_{\bigcirc_{1}, \ldots, \jmath_{n}} \ldots p_{\jmath_{1}, \ldots, \jmath_{k-1}}= \\
& \ldots=p_{\jmath_{1}, \ldots, \jmath_{n}} \ldots p_{\jmath_{1}}=1 \text { and } m_{\jmath_{1}, \ldots, \jmath_{k-1}}>1 .
\end{aligned}
$$

For $z \in \partial D$ define $\varphi_{z}: E \ni \lambda \rightarrow \lambda z \in D$, which is a geodesic in $D$ (see $[\mathrm{JP}])$.

In view of (3.4) there is a point $w \in \partial \mathcal{E}$ such that

$$
w_{\jmath_{1}, \ldots, j_{k}}=0 \text { and } w_{\jmath_{1}, \ldots, j_{k-1}, j_{k}} \neq 0
$$

for $j_{k} \neq j_{k}$. Moreover, there is always at least one $j_{k}$ with this property (by the assumptions on $\mathcal{E}$ and the fact that $m_{\jmath_{1}, \ldots, j_{k-1}}>1$ if $k \geq 2$ ).

Since the extension of $\Phi$ is a homeomorphism between $\bar{D}$ and $\overline{\mathcal{E}}$, there is $\varphi_{z}$ such that $\psi:=\Phi \circ \varphi_{z}$ is a geodesic in $\mathcal{E}$ joining $b$ to $w$ (we now treat $\psi$ as a mapping on $\bar{E}$ ) and even more precisely

$$
\psi(0)=b, \quad \psi(1)=w .
$$

Since (by $(3.3)) \psi_{\jmath_{1}, \ldots, \jmath_{n}} \not \equiv 0$, in view of the form of geodesics in $\mathcal{E}$ (or if some of the components are identically 0 , in some lower dimensional pseudoellipsoid, see Remark 2.3) together with (3.5) and (3.6) we get

$$
\alpha_{\jmath_{1}, \ldots, \jmath_{n}}=1
$$

and for the same reasons,

$$
\text { if } \psi_{\jmath_{1}, \ldots, j_{k}, j_{k+1}, \ldots, j_{n}} \not \equiv 0 \text {, then } \alpha_{\jmath_{1}, \ldots, \jmath_{k}, j_{k+1}, \ldots, j_{n}}=1 \text {. }
$$

We claim that

$$
\left|\alpha_{\jmath_{1}, \ldots, \jmath_{k-1}}\right|<1 \text {. }
$$

Since $\left|\alpha_{0}\right|<1$, we are done in case $k=1$. Suppose that (3.9) does not hold for $k \geq 2$. This means, in view of the form of the geodesics in $\mathcal{E}$ and (3.7), that $\left|\alpha_{j_{1}, \ldots, j_{k-1}}\right|=1$ and consequently for all possible $\left(j_{k}, \ldots, j_{n}\right)$ we get $\alpha_{\jmath_{1}, \ldots, j_{k-1}, j_{k}, \ldots, j_{n}}=1$ or $\psi_{\jmath_{1}, \ldots, \jmath_{k-1}, j_{k}, \ldots, j_{n}} \equiv 0$. This implies that (see $(3.4)$ )

$$
\begin{aligned}
& \psi_{\jmath_{1}, \ldots, \jmath_{k-1}, j_{k}, \ldots, j_{n}}(\lambda) \\
& \quad=C\left(j_{k}, \ldots, j_{n}\right)\left(\frac{1-\lambda}{1-\bar{\alpha}_{\jmath_{1}, \ldots, j_{k-2}} \lambda}\right) \prod_{l=1}^{k-2}\left(\frac{1-\bar{\alpha}_{\jmath_{1}, \ldots, \jmath_{l}} \lambda}{1-\bar{\alpha}_{\rho_{1}, \ldots, \jmath_{l-1}} \lambda}\right),
\end{aligned}
$$

with the product equal to 1 if $k=2$. 
Remark 2.6 together with (3.10) implies that (remember that $\alpha_{0} \in E$ )

$$
w_{\jmath_{1}, \ldots, \jmath_{k-1}, j_{k}, \ldots, j_{n}}=\psi_{\jmath_{1}, \ldots, j_{k-1}, j_{k}, \ldots, j_{n}}(1)=0
$$

for all possible $\left(j_{k}, \ldots, j_{n}\right)$, which, however, contradicts (3.5) and the remark following it.

In view of (3.7), (3.8) and Lemma 3.1 applied repeatedly (if necessary to some lower dimensional pseudoellipsoid) we get

$$
\alpha_{\jmath_{1}, \ldots, j_{k}}=1
$$

Therefore (see (3.4))

$$
\begin{aligned}
& \psi_{\jmath_{1}, \ldots, \jmath_{n}}(\lambda) \\
& =C\left(\frac{1-\lambda}{1-\bar{\alpha}_{\jmath_{1}, \ldots, \jmath_{k-1}} \lambda}\right)^{\frac{1}{p_{\rho_{1}, \ldots, \jmath_{n}} \ldots \rho_{\jmath_{1}, \ldots, \jmath_{k}}}} \prod_{l=1}^{k-1}\left(\frac{1-\bar{\alpha}_{\jmath_{1}, \ldots, \jmath_{l}} \lambda}{1-\bar{\alpha}_{\rho_{1}, \ldots, \jmath_{l-1}} \lambda}\right),
\end{aligned}
$$

with the product equal to 1 if $k=1$.

Since $\varphi_{z}$ extends holomorphically to a neighbourhood of 1 , so does $\psi_{\jmath_{1}, \ldots, \jmath_{n}}$, but since $p_{\jmath_{1}, \ldots, \jmath_{n}} \ldots p_{\jmath_{1}, \ldots, \jmath_{k}}$ is not 1 and is larger than $1 / 2$ we get a contradiction with (3.11). This completes the proof of Proposition 3.2.

Lemma 3.3. Let $\mathcal{E}$ be as in Proposition 3.2. Let $a^{\prime} \in B_{r}$ and $\Psi \in$ Aut $B_{r}$ so that $\Psi\left(a^{\prime}\right)=0$. Define $\Phi: \mathcal{E} \rightarrow \mathbb{C}^{N}$ by setting for $\left(z^{\prime}, w^{\prime}\right) \in \mathcal{E}$, where $z^{\prime}=\left(z_{1}, \ldots, z_{m_{0}}\right) \in \mathbb{C}^{N_{1}}$ and $w^{\prime} \in B_{r}$,

$$
\begin{gathered}
\Phi_{j_{1}, \ldots, j_{n}}\left(z^{\prime}, w^{\prime}\right):=z_{j_{1}, \ldots, j_{n}}\left(\frac{1-\left\|a^{\prime}\right\|^{2}}{\left(1-\left\langle w^{\prime}, a^{\prime}\right\rangle\right)^{2}}\right)^{\frac{1}{2 p_{j_{1}}, \ldots, j_{n} \cdots p_{j_{1}}}}, \\
\Phi_{m_{0}+k}\left(z^{\prime}, w^{\prime}\right):=\Psi_{k}\left(w^{\prime}\right) \quad \text { for } k=1, \ldots, r .
\end{gathered}
$$

Then $\Phi \in$ Aut $\mathcal{E}$.

Proof (as in [JP]). If $r=0$ or $N_{1}=0$, then we are done. In the remaining cases, one can easily see that $\Phi$ is holomorphic and injective. We know that

$$
\begin{aligned}
\left\|\Psi\left(w^{\prime}\right)\right\| & =c_{B_{r}}^{*}\left(0, \Psi\left(w^{\prime}\right)\right) \\
& =c_{B_{r}}^{*}\left(a^{\prime}, w^{\prime}\right)=\left(1-\frac{\left(1-\left\|a^{\prime}\right\|^{2}\right)\left(1-\left\|w^{\prime}\right\|^{2}\right)}{\left|1-\left\langle w^{\prime}, a^{\prime}\right\rangle\right|^{2}}\right)^{1 / 2}
\end{aligned}
$$

for $w^{\prime} \in B_{r}$ (see the formulas for $c_{B_{r}}^{*}$ in $[\mathrm{JP}]$ ).

So for $\left(z^{\prime}, w^{\prime}\right) \in \overline{\mathcal{E}}$ we get 


$$
\begin{aligned}
\sum_{j_{1}=1}^{m_{0}}(\ldots( & \left.\left.\sum_{j_{n}=1}^{m_{j_{1}}, \ldots, j_{n-1}}\left|\Phi_{j_{1}, \ldots, j_{n}}\left(z^{\prime}, w^{\prime}\right)\right|^{2}\right)^{p_{j_{1}, \ldots, j_{n-1}}} \ldots\right)^{p_{j_{1}}} \\
& +\sum_{t=1}^{r}\left|\Phi_{m_{0}+t}\left(z^{\prime}, w^{\prime}\right)\right|^{2} \\
= & -\frac{1-\|\left. a^{\prime}\right|^{2}}{\left|1-\left\langle w^{\prime}, a^{\prime}\right\rangle\right|^{2}} \\
& \times\left(1-\sum_{j_{1}=1}^{m_{0}}\left(\ldots\left(\sum_{j_{n}=1}^{m_{j_{1}, \ldots, j_{n-1}}}\left|z_{j_{1}, \ldots, j_{n}}\right|^{2}\right)^{\left.p_{j_{1}, \ldots, j_{n-1}} \ldots\right)^{p_{j_{1}}}}\right.\right. \\
& \left.-\sum_{t=1}^{r}\left|w_{t}^{\prime}\right|^{2}\right)+1,
\end{aligned}
$$

which finishes the proof.

Cartan's theorem implies that any holomorphic automorphism of $\mathcal{E}$ which preserves the origin is linear. Therefore in view of Proposition 3.2 and Lemma 3.3 we get a description of all holomorphic automorphisms of $\mathcal{E}$.

4. Biholomorphic equivalence of convex complex ellipsoids. In this section we restrict our attention to the case of complex ellipsoids. We write $\mathcal{E}(p):=\left\{\left|z_{1}\right|^{2 p_{1}}+\ldots+\left|z_{n}\right|^{2 p_{n}}<1\right\} \subset \mathbb{C}^{n}$, where $p=\left(p_{1}, \ldots, p_{n}\right)$ with $p_{j}>0$. From now on assume that $n>1$.

In $[\mathrm{JP}]$ the following theorem was proved:

THEOREM 4.1. $\mathcal{E}(p)$ is biholomorphically equivalent to $\mathcal{E}(q)$ iff $p=q$ up to a permutation.

It was also suggested that the proof could be simplified, at least in the convex case, by using the formulas for complex geodesics from Theorem 2.2.

Below we prove this theorem in the convex case utilizing the formulas from Theorem 2.2 and avoiding the use of the theory of the Bergman kernel (as in $[\mathrm{JP}]$ ) or Lie theory (see the results in $[\mathrm{KU}]$ and $[\mathrm{N}]$ ). The key fact in the proof is

ThEOREM 4.2. Let $\Phi: \mathcal{E}(p) \rightarrow \mathcal{E}(q)$ be a biholomorphic mapping, where $p_{j}, q_{j} \geq 1 / 2$ for $j=1, \ldots, n$. Then $\Phi$ extends to a homeomorphism between the closures of the ellipsoids.

This is proved just using the complex geodesics.

It is worth mentioning that it would be desirable to find a theorem analogous to Theorem 2.2 valid for all complex ellipsoids, not necessarily convex. A difficulty arising here is that we do not have just one notion 
of complex geodesic (see Theorem 1.2). Therefore we should try to prove that the mappings defined in Theorem 2.2 describe, in the general case, all $d$-geodesics, where $d$ is $\widetilde{k}, k$ or $c$. If this were the case, then Theorem 4.2 and consequently Theorem 4.1 without the assumption that $p_{j}, q_{j} \geq 1 / 2$ could be proven without any change in the proof. Indeed, the assumption $p_{j}, q_{j} \geq 1 / 2$ is only used in order to show that every biholomorphic mapping maps the image of a mapping as in Theorem 2.2 onto an image of the same form. And this holds for any $d$-geodesics.

Since in the proof of Theorem 4.1 we shall need the explicit formulas for complex geodesics, let us reformulate Theorem 2.2 in the special case of convex ellipsoids in order to have simpler formulas.

TheOREM 2.2' (see [JP] and Theorem 2.2). A bounded holomorphic mapping $\varphi=\left(\varphi_{1}, \ldots, \varphi_{n}\right): E \rightarrow \mathbb{C}^{n}$ is a geodesic for $\mathcal{E}(p)$ with $p_{j} \geq 1 / 2$ if and only if either

$$
\varphi_{j}(\lambda)=a_{j}\left(\frac{\lambda-\alpha_{j}}{1-\bar{\alpha}_{j} \lambda}\right)^{r_{j}}\left(\frac{1-\bar{\alpha}_{j} \lambda}{1-\bar{\alpha}_{0} \lambda}\right)^{1 / p_{j}}
$$

$$
\varphi_{j}(\lambda)=0
$$

where in the case (4.1)

$$
r_{j} \in\{0,1\} \text { and } a_{j} \in \mathbb{C}_{*} \text { for } j=1, \ldots, n, \alpha_{0} \in E,
$$

$\alpha_{j} \in E$ for $j$ such that $r_{j}=1, \alpha_{j} \in \bar{E}$ for $j$ such that $r_{j}=0$,

and (in the case (4.2) we put $\left.\alpha_{j}:=0, a_{j}:=0, r_{j}:=0\right)$,

$$
\alpha_{0}=\sum_{j=1}^{n}\left|a_{j}\right|^{2 p_{j}} \alpha_{j}, \quad 1+\left|\alpha_{0}\right|^{2}=\sum_{j=1}^{n}\left|a_{j}\right|^{2 p_{j}}\left(1+\left|\alpha_{j}\right|^{2}\right),
$$

where the case such that for any $j=1, \ldots, n$ the mapping $\varphi_{j}$ is either of the form (4.2) or of the form (4.1) with $r_{j}=0$ and $\alpha_{j}=\alpha_{0}$ is excluded and the branches of powers are taken so that $1^{1 / p_{j}}=1$.

Before we prove Theorem 4.2 let us formulate and prove the following

LEMMA 4.3. Let $\varphi_{k}, \varphi_{0}: \bar{E} \rightarrow \bar{E}$ for $k=1,2, \ldots$ be mappings of the form

$$
\varphi_{k}(\lambda)=a_{k}\left(\frac{\lambda-\alpha_{k}}{1-\bar{\alpha}_{k} \lambda}\right)^{r_{k}}\left(\frac{1-\bar{\alpha}_{k} \lambda}{1-\bar{\beta}_{k} \lambda}\right)^{1 / m} \quad \text { for } k=0,1, \ldots,
$$

where $m>0, r_{k} \in\{0,1\}, a_{k} \in \mathbb{C}_{*}, \beta_{k} \in E$, and $1^{1 / m}=1, \alpha_{k} \in E$ if $r_{k}=1$, $\alpha_{k} \in \bar{E}$ if $r_{k}=0$. Assume that

$$
\varphi_{k}(0)=\varphi_{0}(0) \quad \text { and } \quad \varphi_{k} \rightarrow \varphi_{0} \text { locally uniformly on } E .
$$

Moreover, if $\varphi_{0}$ is constant (so $r_{0}=0, \alpha_{0}=\beta_{0}$ ), then assume that $\beta_{k} \rightarrow \beta_{0}$. Then $\varphi_{k} \rightarrow \varphi_{0}$ uniformly on $\bar{E}$ and $\beta_{k} \rightarrow \beta_{0}$. 
Proof. First we prove that

$$
\alpha_{k} \rightarrow \alpha_{0}, \quad \beta_{k} \rightarrow \beta_{0},
$$

and, additionally,

(i) if $r_{0}=1$, then $r_{k}=1$ for $k$ sufficiently large and $a_{k} \rightarrow a_{0}$,

(ii) if $r_{0}=0$ and $\left|\alpha_{0}\right|<1$, then $r_{k}=0$ and $a_{k}=a_{0}$ for $k$ sufficiently large,

(iii) if $r_{0}=0,\left|\alpha_{0}\right|=1$ and $r_{k}=0$, then $a_{k}=a_{0}$,

(iv) if $r_{0}=0,\left|\alpha_{0}\right|=1$ and $r_{k}=1$, then $-a_{k} \alpha_{k}=a_{0}$.

Consider case (i). The Hurwitz theorem implies that $\varphi_{k}$ has a root for $k$ sufficiently large. So

$$
\varphi_{k}(\lambda)=a_{k} \frac{\lambda-\alpha_{k}}{1-\bar{\alpha}_{k} \lambda}\left(\frac{1-\bar{\alpha}_{k} \lambda}{1-\bar{\beta}_{k} \lambda}\right)^{1 / m},
$$

with $\alpha_{k} \rightarrow \alpha_{0}$.

Note that

$$
\text { there is } M<\infty \text { such that }\left|a_{k}\right| \leq M \text {, }
$$

otherwise $\varphi_{k}(x)$ would be unbounded for $x \in E$ with $\alpha_{0} \neq x$, which contradicts the fact that $\left|\varphi_{k}(x)\right| \leq 1$.

In view of (4.4),

$$
\varphi_{k}(0)=-a_{k} \alpha_{k}=-a_{0} \alpha_{0} .
$$

In case $\alpha_{0} \neq 0$ this implies that (together with the convergence $\alpha_{k} \rightarrow \alpha_{0}$ ) $a_{k} \rightarrow a_{0}$ and consequently $\beta_{k} \rightarrow \beta_{0}$. So assume that $\alpha_{0}=0$. Suppose that $a_{k}$ does not tend to $a_{0}$ or $\beta_{k}$ does not tend to $\beta_{0}$. Taking subsequences we can assume that $a_{k} \rightarrow x, \beta_{k} \rightarrow y$ and $x \neq a_{0}$ or $y \neq \beta_{0}$ and $|y| \leq 1$ (we may choose such a subsequence because $\left|\beta_{k}\right|<1$ and (4.6)). But this implies that a suitable subsequence

$$
\varphi_{k}(\lambda) \rightarrow \frac{x \lambda}{(1-\bar{y} \lambda)^{1 / m}}
$$

and consequently, in view of (4.4),

$$
\frac{x \lambda}{(1-\bar{y} \lambda)^{1 / m}}=\frac{a_{0} \lambda}{\left(1-\bar{\beta}_{0} \lambda\right)^{1 / m}}
$$

for $\lambda \in E$, which gives $x=a_{0}$ and $y=\beta_{0}$. That gives a contradiction.

Consider now cases (ii)-(iv). Below for brevity we also write $\left(\varphi_{k}\right)$ for some subsequence of $\left(\varphi_{k}\right)$.

Consider the subsequence

$$
\varphi_{k}(\lambda)=a_{k}\left(\frac{1-\bar{\alpha}_{k} \lambda}{1-\bar{\beta}_{k} \lambda}\right)^{1 / m} .
$$


Then in view of (4.4), $a_{k}=a_{0}$ and we get $\alpha_{k} \rightarrow \alpha_{0}$ and $\beta_{k} \rightarrow \beta_{0}$, otherwise we would choose subsequences of $\varphi_{k}$ such that $\alpha_{k} \rightarrow x$ and $\beta_{k} \rightarrow y$ with $y \neq \beta_{0}$ or $x \neq \alpha_{0}$, again leading to a contradiction after making use of the convergence of the subsequence $\varphi_{k}$ (note that if $\varphi_{0}$ is constant, then $x=\alpha_{0}$ and in the other case $\alpha_{0} \neq \beta_{0}$ ).

So we are left with the subsequence

$$
\varphi_{k}(\lambda)=a_{k} \frac{\lambda-\alpha_{k}}{1-\bar{\alpha}_{k} \lambda}\left(\frac{1-\bar{\alpha}_{k} \lambda}{1-\bar{\beta}_{k} \lambda}\right)^{1 / m},
$$

which, in view of $\varphi_{k}(0)=-a_{k} \alpha_{k}=a_{0}=\varphi_{0}(0)$, can be written in the form

$$
\varphi_{k}(\lambda)=a_{0} \frac{1-\frac{1}{\alpha_{k}} \lambda}{1-\bar{\alpha}_{k} \lambda}\left(\frac{1-\bar{\alpha}_{k} \lambda}{1-\bar{\beta}_{k} \lambda}\right)^{1 / m},
$$

First note that $\left|\alpha_{k}\right| \rightarrow 1$. Otherwise we could choose some subsequence of $\varphi_{k}$ such that $\alpha_{k} \rightarrow x$ with $|x|<1$ but in view of (4.8) we get $\varphi_{k}(x) \rightarrow 0$ (remember that $\left|\beta_{k}\right|,\left|\alpha_{k}\right|<1$ ), which contradicts (4.4) (because $\varphi_{0}(x) \neq 0$ ).

We want to prove that $\alpha_{k} \rightarrow \alpha_{0}$ (it will imply that $\left|\alpha_{0}\right|=1$, too). Assume otherwise; then we can assume that for some subsequence $\alpha_{k} \rightarrow$ $x \neq \alpha_{0},|x|=1$ and additionally $\beta_{k} \rightarrow y$. Consequently, in view of (4.8),

$$
\varphi_{k}(\lambda) \rightarrow a_{0} \frac{1-\frac{1}{x} \lambda}{1-\bar{x} \lambda}\left(\frac{1-\bar{x} \lambda}{1-\bar{y} \lambda}\right)^{1 / m}=a_{0}\left(\frac{1-\bar{x} \lambda}{1-\bar{y} \lambda}\right)^{1 / m},
$$

and the limit has to equal

$$
\varphi_{0}(\lambda)=a_{0}\left(\frac{1-\bar{\alpha}_{0} \lambda}{1-\bar{\beta}_{0} \lambda}\right)^{1 / m} .
$$

This proves that $x=\alpha_{0}$ and $y=\beta_{0}$, which settles cases (ii)-(iv).

Before we complete the proof of Lemma 4.3 let us make an auxiliary remark.

Consider the mapping

$$
\Psi: D:=\bar{E} \times \bar{B}(\beta, r) \times \bar{E} \ni\left(\beta_{j}, \beta_{0}, \lambda\right) \rightarrow \frac{1-\bar{\beta}_{j} \lambda}{1-\bar{\beta}_{0} \lambda} \in \mathbb{C},
$$

where $\bar{B}(\beta, r)$ is a closed disk in $\mathbb{C}$ with center $\beta \in E$ and radius $r>0$ such that $\bar{B}(\beta, r) \subset E$.

$\Psi$ is continuous, so $\Psi(D)$ is compact. Since $\operatorname{Re}\left(1-\bar{\beta}_{j} \lambda\right) \geq 0$ and $\operatorname{Re}(1-$ $\left.\bar{\beta}_{0} \lambda\right)>0$, we get $\Psi(D) \subset \mathbb{C} \backslash(-\infty, 0)$.

Together with (4.5) this shows that

$$
\left(\frac{1-\bar{\alpha}_{k} \lambda}{1-\bar{\beta}_{k} \lambda}\right)^{1 / m} \rightarrow\left(\frac{1-\bar{\alpha}_{0} \lambda}{1-\bar{\beta}_{0} \lambda}\right)^{1 / m} \quad \text { uniformly on } \bar{E} \text {. }
$$


Consequently, we get the uniform convergence of $\varphi_{k}$ to $\varphi_{0}$ on $\bar{E}$ if $r_{0}=1$ or $r_{0}=0$ with $\left|\alpha_{0}\right|<1$, and the same convergence of the subsequence of $\varphi_{k}$ such that $r_{k}=0$ if $r_{0}=0$ and $\left|\alpha_{0}\right|=1$. We only need to prove that $\varphi_{k}$ with $r_{k}=1$ tends to $\varphi_{0}$ uniformly on $\bar{E}$, if $r_{0}=0$ and $\left|\alpha_{0}\right|=1$.

We want to prove (see (4.8)) that

$$
a_{0} \frac{1-\frac{1}{\alpha_{k}} \lambda}{1-\bar{\alpha}_{k} \lambda}\left(\frac{1-\bar{\alpha}_{k} \lambda}{1-\bar{\beta}_{k} \lambda}\right)^{1 / m} \rightarrow a_{0}\left(\frac{1-\bar{\alpha}_{0} \lambda}{1-\bar{\beta}_{0} \lambda}\right)^{1 / m}
$$

uniformly on $\bar{E}$.

Set

$$
\begin{array}{ll}
f_{k}(\lambda)=\frac{1-\frac{1}{\alpha_{k}} \lambda}{1-\bar{\alpha}_{k} \lambda}, & g_{k}(\lambda)=\left(\frac{1-\bar{\alpha}_{k} \lambda}{1-\bar{\beta}_{k} \lambda}\right)^{1 / m} \quad \text { for } k=1,2, \ldots, \\
f_{0}(\lambda)=1, & g_{0}(\lambda)=\left(\frac{1-\bar{\alpha}_{0} \lambda}{1-\bar{\beta}_{0} \lambda}\right)^{1 / m} .
\end{array}
$$

In view of (4.9),

$$
g_{k} \rightarrow g_{0} \quad \text { uniformly on } \bar{E} \text {. }
$$

For $1 / 2<\left|\alpha_{k}\right|$ and $|\lambda| \leq 1$ we have

$$
\left|f_{k}(\lambda)-f_{0}(\lambda)\right|=\frac{|\lambda|}{\left|\alpha_{k}\right|} \cdot \frac{1-\left|\alpha_{k}\right|^{2}}{\left|1-\bar{\alpha}_{k} \lambda\right|},
$$

and, consequently,

$$
\left|f_{k}(\lambda)-f_{0}(\lambda)\right| \leq 2 \frac{\left(1-\left|\alpha_{k}\right|\right)\left(1+\left|\alpha_{k}\right|\right)}{1-\left|\alpha_{k}\right||\lambda|}<4 .
$$

To prove (4.10), i.e. that $\left|f_{k} g_{k}-f_{0} g_{0}\right| \rightarrow 0$ uniformly on $\bar{E}$, observe that

$$
\left|f_{k} g_{k}-f_{0} g_{0}\right| \leq\left|f_{k}-f_{0}\right|\left|g_{k}\right|+\left|f_{0}\right|\left|g_{k}-g_{0}\right| \text {. }
$$

In view of (4.12) it is sufficient to show that the first summand in (4.15) is arbitrarily small for $\beta_{k}, \alpha_{k}$ close enough to $\beta_{0}, \alpha_{0}$.

Fix $\varepsilon>0$. There is a neighbourhood $V$ of $\alpha_{0}$ in $\bar{E}$ such that $\left|g_{k}(\lambda)\right|<\varepsilon / 4$ for $\lambda \in V$ (by (4.12), the continuity of $g_{0}$, and the equality $g_{0}\left(\alpha_{0}\right)=0$ ) but $\left\|f_{k}-f_{0}\right\|_{\bar{E}} \leq 4$ (see (4.14)) so on $V$ the first summand of (4.15) behaves well. For $\alpha_{k}, \beta_{k}$ close enough to $\alpha_{0}, \beta_{0}$ we get $\left\|g_{k}\right\|_{\bar{E}} \leq M<\infty$ in view of (4.12). On the other hand, there is $\delta>0$ such that $\left|1-\bar{\alpha}_{k} \lambda\right| \geq \delta$ for $\lambda \in \bar{E} \backslash V$ and for $\alpha_{k}$ close to $\alpha_{0}$. Therefore, if we take $\alpha_{k}$ close enough to $\alpha_{0}$, then in view of (4.13),

$$
\left\|f_{k}-f_{0}\right\|_{\bar{E} \backslash V}<\varepsilon / M
$$

which completes the proof of (4.10) and, consequently, the proof of Lemma 4.3. 
Proof of Theorem 4.2. For any $z \in \partial \mathcal{E}(p)$ define $\varphi_{z}(\lambda):=\lambda z$, which is a complex geodesic. For $z \in \partial \mathcal{E}(p)$ the mapping $\psi_{z}:=\Phi \circ \varphi_{z}$ is a complex geodesic for $\mathcal{E}(q)$. Moreover, in view of Theorem $2.2^{\prime}$, we can extend $\psi_{z}$ continuously to $\bar{E}$. We denote the extension also by $\psi_{z}$. Now we define

$$
\widetilde{\Phi}: \overline{\mathcal{E}(p)} \ni z \rightarrow \begin{cases}\Phi(z) & \text { for } z \in \mathcal{E}(p) \\ \psi_{z}(1) & \text { for } z \in \partial \mathcal{E}(p)\end{cases}
$$

Below we prove that $\widetilde{\Phi}$ is the extension we are looking for.

Note that for $z \in \partial \mathcal{E}(p)$,

$$
\psi_{z}(0)=\Phi(0)=\text { constant. }
$$

Moreover, $\varphi_{z} \rightarrow \varphi_{z_{0}}$ as $z \rightarrow z_{0}, z \in \partial \mathcal{E}(p)$, uniformly on $E$. Consequently, for $z_{0} \in \partial \mathcal{E}(p)$,

$$
\psi_{z} \rightarrow \psi_{z_{0}} \quad \text { as } z \rightarrow z_{0}, z \in \partial \mathcal{E}(p) \text {, locally uniformly on } E \text {. }
$$

We prove more, namely, that for $z_{0} \in \partial \mathcal{E}(p)$,

$$
\psi_{z} \rightarrow \psi_{z_{0}} \quad \text { as } z \rightarrow z_{0}, z \in \partial \mathcal{E}(p) \text {, uniformly on } \bar{E} \text {. }
$$

To prove (4.18) it is sufficient to prove the uniform convergence on $\bar{E}$ of the components of the geodesics. In view of Theorem $2.2^{\prime}$ the components have the form as in Lemma 4.3 (or are identically 0 ), therefore we are done in the cases when $\left(\psi_{z_{0}}\right)_{j}$ is not constant. Take $1 \leq j_{0} \leq n$ such that $\left(\psi_{z_{0}}\right)_{j_{0}} \equiv A$ for some $A \in E$. There is certainly $1 \leq j_{1} \leq n$ such that $\left(\psi_{z_{0}}\right)_{j_{1}}$ is not constant. Since, in view of Theorem $2.2^{\prime}$, we have for $z$ close to $z_{0}$,

$$
\left(\psi_{z}\right)_{j_{1}}(\lambda)=a_{j_{1}, z}\left(\frac{\lambda-\alpha_{j_{1}, z}}{1-\bar{\alpha}_{j_{1}, z} \lambda}\right)^{r_{j_{1}, z}}\left(\frac{1-\bar{\alpha}_{j_{1}, z} \lambda}{1-\bar{\alpha}_{0, z} \lambda}\right)^{1 / q_{j_{1}}}
$$

where $r_{j_{1}, z} \in\{0,1\}$ and the remaining coefficients are as in Theorem 2.2', in view of (4.17) and Lemma 4.3 we get $\alpha_{0, z} \rightarrow \alpha_{0, z_{0}}$ as $z \rightarrow z_{0}, z \in \partial \mathcal{E}(p)$, which implies that

$$
\left|\alpha_{0, z}\right|<\delta<1 \quad \text { for } z \text { close to } z_{0} .
$$

Consider the case $A=0$.

Considering only the points $z \neq z_{0}$ with $\left(\psi_{z}\right)_{j_{0}} \not \equiv 0$ we have as above

$$
\left(\psi_{z}\right)_{j_{0}}(\lambda)=a_{j_{0}, z}\left(\frac{\lambda-\alpha_{j_{0}, z}}{1-\bar{\alpha}_{j_{0}, z} \lambda}\right)^{r_{j_{0}, z}}\left(\frac{1-\bar{\alpha}_{j_{0}, z} \lambda}{1-\bar{\alpha}_{0, z} \lambda}\right)^{1 / q_{j_{0}}} .
$$

We claim that

$$
a_{j_{0}, z} \rightarrow 0 \quad \text { as } z \rightarrow z_{0}, z \in \partial \mathcal{E}(p) .
$$

Note first that $r_{j_{0}, z}=1$, otherwise we would have $a_{j_{0}, z}=0$ (see (4.16)). Also, in view of $(4.16), \alpha_{j_{0}, z}=0$. One easily sees that taking $x \in E_{*}$, the convergence $\left(\psi_{z}(x)\right)_{j_{0}} \rightarrow 0$ implies (4.21). Finally, in view of (4.19), (4.21) and the fact that $\alpha_{j_{0}, z}=0$ we get the desired convergence. 
If $A \neq 0$, then we put

$$
\left(\psi_{z_{0}}(\lambda)\right)_{j_{0}}=a_{j_{0}, z_{0}}\left(\frac{1-\bar{\alpha}_{j_{0}, z_{0}} \lambda}{1-\bar{\alpha}_{0, z_{0}} \lambda}\right)^{1 / q_{j_{0}}} .
$$

Since $\alpha_{0, z} \rightarrow \alpha_{0, z_{0}}$, Lemma 4.3 settles this case.

This completes the proof of (4.18).

Considering neighbourhoods of $z_{0}$ in $\overline{\mathcal{E}(p)}$ of the type

$$
\begin{aligned}
V_{z_{0}}=\left\{\varphi_{z}(t) \text { for } 1 \geq t>s, z \in \partial \mathcal{E}(p),\right. \\
\\
\left.z \text { in some neighbourhood of } z_{0}\right\},
\end{aligned}
$$

for some $s>0$, we see that for any neighbourhood $U$ of $\widetilde{\Phi}\left(z_{0}\right)$ in $\overline{\mathcal{E}(q)}$ there is a set $V$ of the type (4.22) such that $\widetilde{\Phi}(V) \subset U$ (use (4.18)).

Consequently, $\widetilde{\Phi}$ is continuous. Compactness of $\overline{\mathcal{E}(p)}$ and $\overline{\mathcal{E}(q)}$ implies the surjectivity of $\widetilde{\Phi}$.

Applying the same reasoning to $\Phi^{-1}$ we get the continuity of the extension $\Phi^{-1}$. The injectivity of $\widetilde{\Phi}$ (and simultaneously of $\widetilde{\Phi^{-1}}$ ) is a consequence of continuity.

In the proof of Theorem 4.1 we shall need the following technical lemma:

Lemma 4.4. Assume that for some fixed $a, b, c, d \in \mathbb{C}$ and $1 \neq s>0$ we have

$$
\frac{a+b \lambda^{s}}{c+d \lambda^{s}}=\left(\frac{\lambda-\alpha}{1-\bar{\alpha} \lambda}\right)^{t}\left(\frac{1-\bar{\alpha} \lambda}{1-\bar{\beta} \lambda}\right)
$$

for $\lambda$ from some nonempty open set in $E$, where $\alpha \in \bar{E}, \beta \in E, t \geq 0$ and the functions appearing in the equality are not constant. Then $t=s$ and $\alpha=\beta=0$.

Pr o of. Differentiate both sides of the assumed equality, then eliminate the powers of expressions which are not monomials; comparing the coefficients of the powers of $\lambda$ gives the desired result. We omit the tedious but elementary calculations.

Proof of Theorem 4.1 in the convex case. Let $\Phi: \mathcal{E}(p) \rightarrow$ $\mathcal{E}(q)$ be a biholomorphic mapping, which in view of Theorem 4.2 extends to a homeomorphism between the closures of the ellipsoids. Therefore there are open domains $\emptyset \neq U_{1} \subset \mathcal{E}(p), \emptyset \neq U_{2} \subset \mathcal{E}(q)$ such that $U_{1}$ (respectively $U_{2}$ ) is the intersection of some Euclidean ball in $\mathbb{C}^{n}$, not lying entirely in $\mathcal{E}(p)$ (respectively $\mathcal{E}(q)$ ), with $\mathcal{E}(p)$ (respectively $\mathcal{E}(q)$ ), $U_{1}$ and $U_{2}$ do not intersect any axis in the respective ellipsoids and $\Phi\left(U_{1}\right) \subset U_{2}$. Moreover, there are domains $\emptyset \neq \widetilde{U}_{1}, \widetilde{U}_{2} \subset B_{n}$ such that the mappings

(4.23) $z^{p}: U_{1} \ni z \rightarrow\left(z_{1}^{p_{1}}, \ldots, z_{n}^{p_{n}}\right) \in \widetilde{U}_{1}, z^{q}: U_{2} \ni z \rightarrow\left(z_{1}^{q_{1}}, \ldots, z_{n}^{q_{n}}\right) \in \widetilde{U}_{2}$ are biholomorphic. 
Denote the inverse mappings to $z^{p}$ and $z^{q}$ by $z^{1 / p}$ and $z^{1 / q}$ and set

$$
F:=z^{q} \circ \Phi \circ z^{1 / p} \quad \text { on } \widetilde{U}_{1} \text {. }
$$

Then $F$ is a biholomorphic mapping onto the image, which extends to a homeomorphism between the closures such that the extension maps a part of the boundary lying in $\partial B_{n}$ into $\partial B_{n}$.

Rudin's theorem (obtained in $[R]$ with relatively simple tools, owing to which our proof avoids any sophisticated methods) implies that

(4.25) $F$ is a restriction of a holomorphic automorphism of $B_{n}$ to $\widetilde{U}_{1}$, which, in view of (4.24), gives

$$
z^{1 / q} \circ F \circ z^{p}=\Phi \quad \text { on } U_{1} \text {, where } F \in \text { Aut } B_{n} .
$$

Consider now the geodesics (see Theorem $2.2^{\prime}$ ) for $\mathcal{E}(p)$ of the type

$$
\varphi_{\left(j_{0}, A_{1}, \ldots, A_{n}\right)}(\lambda):=\left(A_{1}, \ldots, A_{j_{0}-1}, A_{j_{0}} \lambda, A_{j_{0}+1}, \ldots, A_{n}\right),
$$

where $1 \leq j_{0} \leq n$ and $\left(A_{1}, \ldots, A_{n}\right) \in \partial \mathcal{E}(p)$ is taken from some nonempty open set in $\partial \mathcal{E}(p)$ chosen so that

$$
\varphi_{\left(j_{0}, A_{1}, \ldots, A_{n}\right)}(V) \subset U_{1} \quad \text { for some open set } \emptyset \neq V \subset E \text {. }
$$

Note that $A_{j} \neq 0$ for $j=1, \ldots, n$ and we can take $\left(A_{1}, \ldots, \check{A}_{j_{0}}, \ldots, A_{n}\right)$ from some open set in $\mathcal{E}\left(p_{1}, \ldots, \check{p}_{j_{0}}, \ldots, p_{n}\right)$.

We consider two cases depending on the form of $F$ :

$$
\begin{aligned}
& F=C \circ F_{b}, \quad \text { where } 0 \neq b \in B_{n} \text { and } \\
& \quad F_{b}(z)=\frac{1}{\|b\|^{2}} \cdot \frac{\|b\|^{2}\left(z \sqrt{1-\|b\|^{2}}-b\right)+\langle z, b\rangle b\left(1-\sqrt{1-\|b\|^{2}}\right)}{1-\langle z, b\rangle},
\end{aligned}
$$

where $\langle\cdot, \cdot\rangle$ is the standard scalar product in $\mathbb{C}^{n}$, or

$$
F=C,
$$

where in both cases $C=\left[c_{k l}\right]_{1 \leq k, l \leq n}$ is a unitary mapping.

Below we prove that in both cases the following property holds:

(4.31) if $p_{j_{0}} \neq 1$, then there is $1 \leq k_{0} \leq n$ such that $q_{k_{0}}=p_{j_{0}}$; for $j_{0} \neq j_{1}$ with $p_{j_{0}}=p_{j_{1}} \neq 1$ there are $1 \leq k_{0}, k_{1} \leq n$ such that $k_{0} \neq k_{1}$ and $q_{k_{0}}=q_{k_{1}}=p_{j_{0}}$.

Note that if we assume (4.31), then applying the same reasoning to $\Phi^{-1}$ we get $p=q$, up to a permutation.

Therefore, it is sufficient to prove (4.31) in the cases (4.29) and (4.30).

In view of (4.26) we have

$$
F \circ\left(\varphi_{\left(j_{0}, A_{1}, \ldots, A_{n}\right)}(\lambda)^{p}\right)=z^{q} \circ \Phi\left(\varphi_{\left(j_{0}, A_{1}, \ldots, A_{n}\right)}(\lambda)\right) \quad \text { for } \lambda \in V .
$$

Fix $j_{0}$ such that $p_{j_{0}} \neq 1$. 
In the case (4.29), for $j \neq j_{0}$,

$$
\begin{aligned}
\left(F_{b}\left(\varphi_{\left(j_{0}, A_{1}, \ldots, A_{n}\right)}(\lambda)^{p}\right)\right)_{j} & \\
= & \frac{1}{\|b\|^{2}}\left(\frac{\left\|b^{2}\right\|\left(A_{j}^{p_{j}} \sqrt{1-\|b\|^{2}}-b_{j}\right)}{1-\sum_{l \neq j_{0}} A_{l}^{p_{l}} \bar{b}_{l}-A_{j_{0}}^{p_{0}} \bar{b}_{j_{0}} \lambda^{p_{j_{0}}}}\right. \\
& \left.+\frac{\left(\sum_{l \neq j_{0}} A_{l}^{p_{l}} \bar{b}_{l}+A_{j_{0}}^{p_{j_{0}}} \bar{b}_{j_{0}} \lambda^{p_{j_{0}}}\right) b_{j}\left(1-\sqrt{1-\|b\|^{2}}\right)}{1-\sum_{l \neq j_{0}} A_{l}^{p_{l}} \bar{b}_{l}-A_{j_{0}}^{p_{j_{0}}} \bar{b}_{j_{0}} \lambda^{p_{j_{0}}}}\right)
\end{aligned}
$$

and

$$
\begin{aligned}
& \left(F_{b}\left(\varphi_{\left(j_{0}, A_{1}, \ldots, A_{n}\right)}(\lambda)^{p}\right)\right)_{j_{0}} \\
& =\frac{1}{\|b\|^{2}}\left(\frac{\left\|b^{2}\right\|\left(A_{j_{0}}^{p_{j_{0}}} \lambda^{p_{j_{0}}} \sqrt{1-\|b\|^{2}}-b_{j_{0}}\right)}{1-\sum_{l \neq j_{0}} A_{l}^{p_{l}} \bar{b}_{l}-A_{j_{0}}^{p_{j_{0}}} \bar{b}_{j_{0}} \lambda_{j_{0}}^{p_{j_{0}}}}\right) \\
& \left.\quad+\frac{\left(\sum_{l \neq j_{0}} A_{l}^{p_{l}} \bar{b}_{l}+A_{j_{0}}^{p_{j_{0}}} \bar{b}_{j_{0}} \lambda^{p_{j_{0}}}\right) b_{j_{0}}\left(1-\sqrt{1-\|b\| \|^{2}}\right)}{1-\sum_{l \neq j_{0}} A_{l}^{p_{l}} \bar{b}_{l}-A_{j_{0}}^{p_{j_{0}}} \bar{b}_{j_{0}} \lambda^{p_{j_{0}}}}\right) .
\end{aligned}
$$

Consequently, for $k=1, \ldots, n$ we have

$$
\left(F\left(\varphi_{\left(j_{0}, A_{1}, \ldots, A_{n}\right)}(\lambda)^{p}\right)\right)_{k}=\frac{1}{\|b\|^{2}} \cdot \frac{D_{1} \lambda^{p_{j}}+D_{2}}{1-\sum_{l \neq j_{0}} A_{l}^{p_{l}} \bar{b}_{l}-A_{j_{0}}^{p_{j_{0}}} \bar{b}_{j_{0}} \lambda^{p_{j_{0}}}}
$$

where

$$
\begin{aligned}
D_{1}= & \left(\sum_{j} c_{k j} b_{j}\right)\left(A_{j_{0}}^{p_{j}} \bar{b}_{j_{0}}\left(1-\sqrt{1-\|b\|^{2}}\right)\right)+c_{k j_{0}}\|b\|^{2} A_{j_{0}}^{p_{j_{0}}} \sqrt{1-\|b\|^{2}}, \\
D_{2}= & \|b\|^{2} \sum_{j \neq j_{0}} c_{k j}\left(A_{j}^{p_{j}} \sqrt{1-\|b\|^{2}}-b_{j}\right)-c_{k j_{0}}\|b\|^{2} b_{j_{0}} \\
& +\left(\sum_{l \neq j_{0}} A_{l}^{p_{l}} \bar{b}_{l}\right) \sum_{j} b_{j} c_{k j}\left(1-\sqrt{1-\|b\|^{2}}\right) .
\end{aligned}
$$

The properties of $\varphi_{\left(j_{0}, A_{1}, \ldots, A_{n}\right)}$ and Theorem 2.2' imply that $\Phi \circ$ $\varphi_{\left(j_{0}, A_{1}, \ldots, A_{n}\right)}$ is a complex geodesic of the form

$$
\left(\Phi \circ \varphi_{\left(j_{0}, A_{1}, \ldots, A_{n}\right)}\right)_{k}^{q_{k}}(\lambda)=a_{k}^{q_{k}}\left(\frac{\lambda-\alpha_{k}}{1-\bar{\alpha}_{k} \lambda}\right)^{r_{k} q_{k}}\left(\frac{1-\bar{\alpha}_{k} \lambda}{1-\bar{\alpha}_{0} \lambda}\right),
$$

where $r_{k}, \alpha_{k}, \alpha_{0}, a_{k}$ have the properties as in Theorem $2.2^{\prime}$.

Comparing the expressions and the exponents in (4.35) and (4.36) we see in view of Lemma 4.4 that $\left(\Phi \circ \varphi_{\left(j_{0}, A_{1}, \ldots, A_{n}\right)}\right)_{k}^{q_{k}}$ can be nonconstant only for $k$ such that

$$
q_{k}=p_{j_{0}} \quad \text { with } r_{k}=1, \alpha_{k}=\alpha_{0}=0 .
$$

We then have $b_{j_{0}}=0$ and also 
(4.38) $\quad D_{1} \neq 0$ and $D_{2}=0$ for $k$ such that $\left(\Phi \circ \varphi_{\left(j_{0}, A_{1}, \ldots, A_{n}\right)}\right)_{k}^{q_{k}}$ is not constant, $D_{1}=0$ for $k$ such that $q_{k} \neq p_{j_{0}}$.

From (4.38) we conclude that

(4.39) $\quad c_{k j_{0}} \neq 0$ for $k$ with $\left(\Phi \circ \varphi_{\left(j_{0}, A_{1}, \ldots, A_{n}\right)}\right)_{k}^{q_{k}}$ nonconstant and $c_{k j_{0}}=0$ for $k$ with $q_{k} \neq p_{j_{0}}$.

Hence for any $j$ with $p_{j} \neq 1$ we have

$$
b_{j}=0 \quad \text { and } \quad c_{k j}=0 \quad \text { whenever } q_{k} \neq p_{j} .
$$

Since $F \circ \varphi_{\left(j_{0}, A_{1}, \ldots, A_{n}\right)}$ is not a constant mapping for any $\left(A_{1}, \ldots, A_{n}\right)$, there is $k_{0}$ such that $\left(\Phi \circ \varphi_{\left(j_{0}, A_{1}, \ldots, A_{n}\right)}\right)_{k_{0}}^{q_{k_{0}}}$ is not constant for $\left(A_{1}, \ldots\right.$, $\left.\check{A}_{j_{0}}, \ldots, A_{n}\right)$ from some open set in $\left.\mathcal{E}\left(p_{1}, \ldots, \check{p}_{j_{0}}, \ldots, p_{n}\right)\right)$. Then (4.38) implies that

$$
\begin{aligned}
\sum_{j \neq j_{0}} c_{k_{0} j}\left(\|b\|^{2}\left(A_{j}^{p_{j}} \sqrt{1-\|b\|^{2}}-b_{j}\right)\right. & \\
& \left.+\left(\sum_{l \neq j_{0}} A_{l}^{p_{l}} \bar{b}_{l}\right) b_{j}\left(1-\sqrt{1-\|b\|^{2}}\right)\right)=0
\end{aligned}
$$

for $\left(A_{1}, \ldots, \check{A}_{j_{0}}, \ldots, A_{n}\right)$ from an open set. (4.41) is in view of (4.40) equivalent to

$$
\begin{aligned}
& \sum_{\substack{j \neq j_{0} \\
q_{k_{0}}=p_{j} \neq 1}} c_{k_{0} j}\|b\|^{2} A_{j}^{p_{j}} \sqrt{1-\|b\|^{2}} \\
& +\sum_{\substack{j \neq j_{0} \\
p_{j}=1}} c_{k_{0} j}\left(\|b\|^{2}\left(A_{j} \sqrt{1-\|b\|^{2}}-b_{j}\right)\right. \\
& \left.+\left(\sum_{\substack{l \neq j_{0} \\
p_{l}=1}} A_{l} \bar{b}_{l}\right) b_{j}\left(1-\sqrt{1-\|b\|^{2}}\right)\right)=0
\end{aligned}
$$

for the numbers $A_{j}$ as above, which implies that

$$
\sum_{\substack{j \neq j_{0} \\ q_{k_{0}}=p_{j} \neq 1}} c_{k_{0} j}\|b\|^{2} A_{j}^{p_{j}} \sqrt{1-\|b\|^{2}}=\text { constant }
$$

for $0 \neq A_{j}^{p_{j}}$ with $p_{j}=q_{k_{0}}, j \neq j_{0}$, taken from some nonempty set.

Therefore

$$
c_{k_{0} j_{0}} \neq 0 \text { and } \quad c_{k_{0} j}=0 \text { for } j \neq j_{0} \text { with } q_{k_{0}}=p_{j}=p_{j_{0}} .
$$

For $j_{0} \neq j_{1}$ with $p_{j_{0}}=p_{j_{1}} \neq 1$ we see, in view of (4.42), that there are $k_{0} \neq k_{1}$ such that $q_{k_{0}}=q_{k_{1}}=p_{j_{0}}$.

This completes the case (4.31). 
In the case (4.30) we have

$$
\left(F\left(\varphi_{\left(j_{0}, A_{1}, \ldots, A_{n}\right)}(\lambda)^{p}\right)\right)_{k}=\sum_{j \neq j_{0}} c_{k j} A_{j}^{p_{j}}+c_{k j_{0}} A_{j_{0}}^{p_{j_{0}}} \lambda^{p_{j_{0}}} .
$$

As before (4.43) has to equal (4.36), which implies that (see Lemma 4.4) (4.43) is not constant only for $k$ such that

$$
q_{k}=p_{j_{0}} \quad \text { and } \quad r_{k}=1, \alpha_{k}=\alpha_{0}=0 .
$$

Take $k_{0}$ as before such that $(4.43)$ is not constant for $\left(A_{1}, \ldots, \check{A}_{j_{0}}, \ldots\right.$ $\left.\ldots, A_{n}\right)$ from a nonempty open set in $\mathcal{E}\left(p_{1}, \ldots, \check{p}_{j_{0}}, \ldots, p_{n}\right)$. Then we easily conclude that

$$
\sum_{j \neq j_{0}} c_{k_{0} j} A_{j}^{p_{j}}=0
$$

which implies that $c_{k_{0} j}=0$ for $j \neq j_{0}$, so $c_{k_{0} j_{0}} \neq 0$, which in view of the fact that $C$ is unitary completes the proof of (4.31).

Acknowledgments. The author would like to express his gratitude to Professor Peter Pflug for his precious remarks and hints concerning the paper.

\section{References}

[B] S. Bell, The Bergman kernel function and proper holomorphic mappings, Trans. Amer. Math. Soc. 270 (1983), 685-691.

[BFKKMP] B. E. Blank, D. Fan, D. Klein, S. G. Krantz, D. Ma and M.-Y. Pang, The Kobayashi metric of a complex ellipsoid in $\mathbb{C}^{2}$, Experimental Math. 1 (1992), 47-55.

[D] S. Dineen, The Schwarz Lemma, Clarendon Press, 1989.

[DT] S. Dineen and R. M. Timoney, Complex geodesics on convex domains, in: Progress in Functional Analysis, K. D. Bierstedt, J. Bonet, J. Horváth and M. Maestre (eds.), Elsevier, 1992, 333-365.

[DP] G. Dini and A. S. Primicerio, Localization principle of automorphisms on generalized pseudoellipsoids, preprint.

[Ga] J. Garnett, Bounded Analytic Functions, Academic Press, New York, 1981.

[Ge] G. Gentili, Regular complex geodesics in the domain $D_{n}=\left\{\left(z_{1}, \ldots, z_{n}\right) \in\right.$ $\left.\mathbb{C}^{n}:\left|z_{1}\right|+\ldots+\left|z_{n}\right|<1\right\}$, in: Lecture Notes in Math. 1275, Springer, 1987, 235-252.

[JP] M. Jarnicki and P. Pflug, Invariant Distances and Metrics in Complex Analysis, Walter de Gruyter, 1993.

[JPZ] M. Jarnicki, P. Pflug and R. Zeinstra, Geodesics for convex complex ellipsoids, Ann. Scuola Norm. Sup. Pisa 20 (1993), 535-543.

[KU] W. Kaup and H. Upmeier, Banach spaces with biholomorphically equivalent unit balls are isomorphic, Proc. Amer. Math. Soc. 58 (1976), 129-133. 
[KKM] A. Kodama, S. Krantz and D. Ma, A characterization of generalized complex ellipsoids in $\mathbb{C}^{n}$ and related results, Indiana Univ. Math. J. 41 (1992), 173-195.

[L] L. Lempert, La métrique de Kobayashi et la représentation des domaines sur la boule, Bull. Soc. Math. France 109 (1981), 427-479.

[N] T. Naruki, The holomorphic equivalence problem for a class of Reinhardt domains, Publ. RIMS Kyoto Univ. 4 (1968), 527-543.

[P] E. A. Poletskii, The Euler-Lagrange equations for extremal holomorphic mappings of the unit disk, Michigan Math. J. 30 (1983), 317-333.

[R] W. Rudin, Holomorphic maps that extend to automorphisms of a ball, Proc. Amer. Math. Soc. 81 (1981), 429-432.

INSTITUTE OF MATHEMATICS

JAGIELLONIAN UNIVERSITY

REYMONTA 4

30-059 KRAKÓW, POLAND

Reçu par la Rédaction le 16.6.1994

Révisé le 2\%.2.1995 